\title{
sciendo
}

\author{
BULGARIAN ACADEMY OF SCIENCES
}

CYBERNETICS AND INFORMATION TECHNOLOGIES • Volume 20, No 5

Special issue on Innovations in Intelligent Systems and Applications

Sofia $\bullet 2020$

Print ISSN: 1311-9702; Online ISSN: 1314-4081

DOI: $10.2478 /$ cait-2020-0039

\section{Two Applications of Inter-Criteria Analysis with Belief Functions}

\author{
Jean Dezert ${ }^{1}$, Albena Tchamova ${ }^{2}$, Stefka Fidanova ${ }^{2}$, Deqiang Han ${ }^{3}$ \\ ${ }^{1}$ The French Aerospace Lab, Palaiseau, France \\ ${ }^{2}$ Institute of Information and Communication Technologies, Bulgarian Academy of Sciences, 1113 Sofia, \\ Bulgaria \\ ${ }^{3}$ Institute of Integrated Automation Xi'an Jiaotong University, China \\ E-mails: jean.dezert@onera.fr tchamova@bas.bg stefka@parallel.bas.bg deqhan@gmail.com
}

\begin{abstract}
In this paper we present two applications of a new Belief Function-based InterCriteria Analysis (BF-ICrA) approach for the assessment of redundancy of criteria involved in Multi-Criteria Decision-Making (MCDM) problems. This BF-ICrA method allows to simplify the original MCDM problem by suppressing redundant criteria (if any) and thus diminish the complexity of MCDM problem. This approach is appealing for solving large MCDM problems whose solution requires the fusion of many belief functions. We show how this approach can be used in two distinct fields of applications: The GPS surveying problem, and the car selection problem.
\end{abstract}

Keywords: Inter-Criteria Analysis, ICrA-BF, MultiCriteria Decision Making, MCDM, belieffunctions.

\section{Introduction}

In a Multi-Criteria Decision-Making (MCDM) problem we consider a set of alternatives (or objects) $A \triangleq\left\{A_{1}, A_{2}, \ldots, A_{M}\right\} \quad(M>2)$ and a set of criteria $C \triangleq\left\{C_{1}, C_{2}, \ldots, C_{N}\right\}(N \geq 1)$. We search for the best alternative $A^{*}$ given the available information expressed by a $M \times N$ score matrix (also called benefit or payoff matrix) $S \triangleq\left[S_{i j}=C_{j}\left(A_{i}\right)\right]$, and (eventually) the importance factor $\omega_{j} \in[0,1]$ of each criterion $C_{j}$ with $\sum_{j=1}^{N} \omega_{j}=1$. The set of normalized weighting factors is denoted by $\omega \triangleq\left\{\omega_{1}, \omega_{2}, \ldots, \omega_{N}\right\}$. Depending on the context of the MCDM problem, the score $S_{i j}$ of each alternative $A_{i}$ with respect to each criteria $C_{j}$ can be interpreted either as a cost (i.e., an expense), or as a reward (i.e., a benefit). By convention and without loss of generality (Because it suffices to multiply the scores values by -1 to reverse the preference ordering) we will always interpret the score as a reward having monotonically increasing preference. Thus, the best alternative $A_{j}^{*}$ for a given criteria $C_{j}$ will be the one providing the highest reward/benefit. 
The MCDM problem is not easy to solve because the scores are usually expressed in different (physical) units and different scales. This necessitates a choice of score/data normalization yielding rank reversal problems [1,2]. Usually there is no same best alternative choice $A^{*}$ for all criteria, so a compromise has to be established to provide a reasonable and acceptable solution of the MCDM problem for decision-making support.

Many MCDM methods exist, see references in [3]. Most popular methods are AHP (Analytic Hierarchy Process) [4] ELECTRE (ELimination Et Choix Traduisant la REalité) [5], TOPSIS (Technique for Order Preference by Similarity to Ideal Solution) [6, 7]. In 2016 and 2017, we did develop BF-TOPSIS methods [3, 8] based on Belief Functions (BF) to improve the original TOPSIS approach to avoid data normalization and to deal also with imprecise score values as well. It appears however that the complexity of these new BF-TOPSIS methods can become a bottleneck for their use in large MCDM problems due to the fusion step of basic belief assignments required for the implementation of the BF-TOPSIS. That is why a simplification of the MCDM problem (if possible) is very welcome in order to save computational time and resources. This is the motivation of the present work.

For this aim we propose a new Inter-Criteria Analysis (ICrA) based on belief functions for identifying and estimating the possible degree of agreement (i.e., redundancy) between some criteria driven from the data (score values). This permits to remove all redundant criteria of the original MCDM problem and thus solving a simplified (almost) equivalent MCDM problem faster and at lower computational cost. ICrA has been developed originally by Atanassov, Mavrov and Atanassova [9], Atanassov, Atanassova and Gluhchev [10] and A t a n a s o v et al. [11], based on Intuitionistic Fuzzy Sets [12], and it has been applied in different fields like medicine [13-15], optimization [16-20] workforce planning [21], competitiveness analysis [22], radar detection [23], ranking [24-27], etc. In this paper we improve ICrA approach thanks to belief functions introduced by Shafer in [28] from original Dempster's works [29]. We will refer it as BF-ICrA method in the sequel.

After a short presentation of basics of belief functions in Section 2, we present Atanassov's ICrA method in Section 3 and discuss its limitations. In Section 4 we present the new BF-ICrA approach based on a new construction of Basic Belief Assignment (BBA) matrix from the score matrix and a new establishment of InterCriteria belief matrix. In Section 5 a method of simplification of MCDM using BFICrA is proposed. Two distinct applications of BF-ICrA are presented in Section 6 with concluding remarks in Section 7.

\section{Basics of the theory of belief functions}

To follow classical notations of the theory of belief functions, also called DempsterShafer Theory (DST) [28], we assume that the answer (i.e., the solution, or the decision to take) of the problem under concern belongs to a known finite discrete frame of discernments (FoD) $\Theta \triangleq\left\{\theta_{1}, \theta_{2}, \ldots, \theta_{n}\right\}$ with $n>1$, and where all elements of $\Theta$ are exclusive. The set of all subsets of $\Theta$ (including empty set $\emptyset$ and $\Theta$ ) is the 
power-set of $\Theta$ denoted by $2^{\Theta}$. A BBA (or mass function) associated with a given source of evidence is defined in [28] as the mapping $m(\cdot): 2^{\Theta} \rightarrow[0,1]$ satisfying $m(\varnothing)=0$ and $\sum_{A \in 2^{\Theta}} m(A)=1$. The quantity $m(A)$ is called the mass of $A$ committed by the source of evidence. Belief and plausibility functions are usually interpreted respectively as lower and upper bounds of unknown (possibly subjective) probability measure [29]. They are defined by

$$
\operatorname{Bel}(A) \triangleq \sum_{B \subseteq A, B \in 2^{\Theta}} m(B) \text { and } \operatorname{Pl}(A) \triangleq 1-\operatorname{Bel}(\bar{A}),
$$

where the symbol $\triangleq$ means equal by definition.

If $m(A)>0, A$ is called a focal element of $m(\cdot)$. When all focal elements are singletons then $m(\cdot)$ is called Bayesian BBA [28] and its corresponding Bel(·) function is homogeneous to a probability measure. The vacuous BBA, or VBBA for short, representing a totally ignorant source is defined as $m_{\vartheta}(\Theta)=1$. The main challenge of the decision-maker consists to combine efficiently the possible multiple BBAs $m_{s}(\cdot)$ given by $s>1$ distinct sources of evidence to obtain a global (combined) BBA, and to make a final decision from it. Historically the combination of BBAs is accomplished by Dempster's rule proposed by Shafer in DST. Because Dempster's rule presents several serious problems (insensitivity to the level of conflict between sources in some cases, inconsistency with bounds of conditional probabilities when used for belief conditioning, dictatorial behaviour, counterintuitive results), many fusion rules have been proposed in the literature as alternative to Dempster's rule, see [30, Vol. 2] for a detailed list of fusion rules. We will not detail here all the possible combination rules but just mention that the Proportional Conflict Redistribution rule No 6 (PCR6) proposed by Martin and Osswald in [30] (Vol. 3) is one of the most serious alternative rule for BBA combination available so far.

\section{Atanassov's Inter-Criteria Analysis (ICrA)}

ICrA approach is based on a $M \times N$ score matrix $S \triangleq\left[S_{i j}=C_{j}\left(A_{i}\right), i=1, \ldots, M\right.$, $j=1, \ldots, N]$ (index matrix by Atanassov in [31]), and intuitionistic fuzzy pairs [12] including two membership functions $\mu(\cdot)$ and $v(\cdot)$. Mathematically, an Intuitionistic Fuzzy Set (IFS) $A$ is denoted by $A \triangleq\left\{\left(x, \mu_{A}(x), \vartheta_{A}(x)\right) \mid x \in E\right\}$ where $E$ is the set of possible values of $x$ to the set $A, \mu_{A}(x) \in[0,1]$ defines the membership of $x$ to the set $A$, and $\vartheta_{A}(x) \in[0,1]$ defines the non-membership of $x$ to the set $A$ with the restriction $0 \leq \mu_{A}(x)+\vartheta_{A}(x) \leq 1$. The ICrA method consists in building an $N \times N$ Inter-Criteria (IC) matrix from the score matrix $S$. The elements of the IC matrix consist of all intuitionistic fuzzy pairs $\left(\mu_{j j^{\prime}}, \vartheta_{j j^{\prime}}\right)$ whose components express respectively the degree of agreement and the degree of disagreement between criteria $C_{j}$ and $C_{j^{\prime}}$ for $j, j^{\prime} \in\{1,2, \ldots, N\}$. For a given column $j$ (i.e., criterion $C_{j}$ ), it is always possible to compare with $>,<$ and $=$ operators all the scores $S_{i j}$ for $i=1,2, \ldots, M$ because the scores of each column are expressed in the same unit. The construction of IC matrix can be used to search relations between the criteria because the method compares homogeneous data relatively to a same column. A t a n a s s o v et al. [32] prescribes to normalize the score matrix before applying ICrA as follows: 


$$
S_{i j}^{\text {norm }}=\frac{S_{i j}-S_{j}^{\min }}{S_{j}^{\max }-S_{j}^{\min }}
$$

In our new Belief Function based ICrA approach it is not necessary to apply a score matrix normalization because each column of the score matrix represents the values of one and the same criterion for different alternatives, and the criterion values are expressed with the same unit (e.g., $\mathrm{m}, \mathrm{m}^{2}, \mathrm{~s}, \mathrm{~kg}$, or $€$, etc.) from which belief functions are established without regards to the specific unit of each criterion.

\subsection{Construction of inter-criteria matrix}

The construction of the $N \times N$ IC matrix, denoted by $\mathbf{K}$ is based on the pairwise comparisons between every two criteria along all evaluated alternatives. We use $\mathbf{K}$ because it corresponds to the first letter of word Kriterium, meaning criteria in German. The letter $\mathbf{C}$ is already used.

Let $K_{j j^{\prime}}^{\vartheta}$ be the number of cases in which the inequalities $S_{i j}>S_{i^{\prime} j}$ and $S_{i j^{\prime}}>S_{i^{\prime} j^{\prime}}$ hold simultaneously, and let $K_{j j^{\prime}}^{\vartheta}$ be the number of cases in which the inequalities $S_{i j}>S_{i^{\prime} j}$ and $S_{i j^{\prime}}<S_{i^{\prime} j^{\prime}}$ hold simultaneously. Because the total number of comparisons between the alternatives is $M(M-1) / 2$ then one always has necessarily

$$
0 \leq K_{j j^{\prime}}^{\mu}+K_{j j^{\prime}}^{\vartheta} \leq \frac{M(M-1)}{2},
$$

or equivalently after the division by $\frac{M(M-1)}{2}>0$,

$$
0 \leq \frac{2 K_{j j^{\prime}}^{\mu}}{M(M-1)}+\frac{2 K_{j j^{\prime}}^{\vartheta}}{M(M-1)} \leq 1 .
$$

This inequality permits to define the elements of $N \times N$ IC matrix $\mathbf{K}=\left[K_{j j^{\prime}}\right]$ as Intuitionistic Fuzzy (IF) pairs $K_{j j^{\prime}}=\left(\mu_{j j^{\prime}}, \vartheta_{j j^{\prime}}\right)$ where

$$
\mu_{j j^{\prime}} \triangleq \frac{2 K_{j j^{\prime}}^{\mu}}{M(M-1)} \text {, and } \vartheta_{j j^{\prime}} \triangleq \frac{2 K_{j j^{\prime}}^{\vartheta}}{M(M-1)^{\prime}}
$$

where $\mu_{j j^{\prime}}$ measures the degree of agreement between criteria $C_{j}$ and $C_{j^{\prime}}$, and $\vartheta_{j j^{\prime}}$ measures their degree of disagreement. By construction the IC matrix $\mathbf{K}$ is always a symmetric matrix. The computation of $K_{j j^{\prime}}^{\mu}$ and $K_{j j^{\prime}}^{\vartheta}$ can be done explicitly thanks to Atanassov's formulas [32]

and

$$
\begin{gathered}
K_{j j^{\prime}}^{\mu}=\sum_{i=1}^{M-1} \sum_{i^{\prime}=i+1}^{M}\left[\operatorname{sgn}\left(S_{i j}-S_{i^{\prime} j}\right) \operatorname{sgn}\left(S_{i j^{\prime}}-S_{i^{\prime} j^{\prime}}\right)+\right. \\
\left.+\operatorname{sgn}\left(S_{i^{\prime} j}-S_{i j}\right) \operatorname{sgn}\left(S_{i^{\prime} j^{\prime}}-S_{i j^{\prime}}\right)\right]
\end{gathered}
$$

$$
\begin{gathered}
K_{j j^{\prime}}^{v}=\sum_{i=1}^{M-1} \sum_{i^{\prime}=i+1}^{M}\left[\operatorname{sgn}\left(S_{i j}-S_{i^{\prime} j}\right) \operatorname{sgn}\left(S_{i^{\prime} j^{\prime}}-S_{i j^{\prime}}\right)+\right. \\
\left.+\operatorname{sgn}\left(S_{i^{\prime} j}-S_{i j}\right) \operatorname{sgn}\left(S_{i j^{\prime}}-S_{i^{\prime} j^{\prime}}\right)\right],
\end{gathered}
$$

where the signum function sgn(.) used by Atanassov is defined as

$$
\operatorname{sgn}(x)= \begin{cases}1 & \text { if } x>0 \\ 0 & \text { if } x \leq 0\end{cases}
$$

Actually the values of $K_{j j^{\prime}}^{\mu}$ and $K_{j j^{\prime}}^{\vartheta}$ depend on the choice of $\operatorname{sgn}(x)$ function. For instance if we use $\operatorname{sqn}(x)=1$ if $x \geq 0$ and $\operatorname{sqn}(x)=0$ if $x<0$ we will 
obtain, in general, other $K_{j j^{\prime}}^{\mu}$ and $K_{j j^{\prime}}^{\vartheta}$ values. That is why in $[21,33]$ the authors propose different algorithms implemented under Java in an ICrA software yielding different $K_{j j^{\prime}}^{\mu}$ and $K_{j j^{\prime}}^{\vartheta}$ values for making the analysis and to reduce the dimension (complexity) of the original MCDM problem.

\subsection{Inter-criteria analysis}

Once the Inter-Criteria matrix $K=\left[K_{j j^{\prime}}\right]$ of intuitionistic fuzzy pairs is calculated one needs to analyse it to decide which criteria $C_{j}$ and $C_{j^{\prime}}$ are in strong agreement (or positive consonance) reflecting the correlation between $C_{j}$ and $C_{j^{\prime}}$ in strong disagreement (or negative consonance) reflecting non correlation between $C_{j}$ and $C_{j^{\prime}}$, or in dissonance reflecting the uncertainty situation where nothing can be said about the non-correlation or the correlation between $C_{j}$ and $C_{j^{\prime}}$. If one wants to identify the set of criteria $C_{j^{\prime}}$ for $j^{\prime} \neq j$ that are strongly correlated with $C_{j}$ then we can sort $\mu_{j j^{\prime}}$ values is descending order to identify those in strong positive consonance with $C_{j}$. In $[25,26]$ the authors propose a qualitative scale to refine the levels of consonance and dissonance and for helping the decision making procedure. A dual approach based on $\vartheta_{j j^{\prime}}$ values can be made to determine the set of criteria that are not correlated with $C_{j}$. Another approach [10,27] proposes to define two thresholds $\alpha, \beta \in[0,1]$ for the positive and negative consonance respectively against which the components $\mu_{j j^{\prime}}$ and $\vartheta_{j j^{\prime}}$ of $K_{j j^{\prime}}=\left(\mu_{j j^{\prime}}, \vartheta_{j j^{\prime}}\right)$ will be compared. The correlations between the criteria $C_{j}$ and $C_{j^{\prime}}$ are called "positive consonance", "negative consonance" or "dissonance" depending on their $\mu_{j j^{\prime}}$ and $\vartheta_{j j^{\prime}}$ values with respect to chosen thresholds $\alpha$ and $\beta$, see [22] for details. More precisely, $C_{j}$ and $C_{j^{\prime}}$ are in

- $(\alpha, \beta)$ positive consonance (i.e., correlated):

$$
\text { If } \mu_{j j^{\prime}}>\alpha \text { and } \vartheta_{j j^{\prime}}<\beta \text {, }
$$

- $(\alpha, \beta)$ negative consonance (i.e., no correlated):

$$
\text { If } \mu_{j j^{\prime}}<\beta \text { and } \vartheta_{j j^{\prime}}>\alpha \text {, }
$$

- $(\alpha, \beta)$ dissonance (i.e., full uncertainty) otherwise.

At the beginning of ICrA development it was not very clear how these Intuitionistic Fuzzy (IF) pairs $\left(\mu_{j j^{\prime}}, \vartheta_{j j^{\prime}}\right)$ had to be used and that is why Atanassova $[34,35]$ proposed to handle both components of the IF pair. For this, she interpreted pairs $=\left(\mu_{j j^{\prime}}, \vartheta_{j j^{\prime}}\right)$ as points located in the elementary TFU triangle, where the point $T$ of coordinate $(1,0)$ represents the maximal positive consonance (i.e., the true consonance), the point $F$ with coordinate $(0,1)$ represents the maximal negative consonance (i.e., the falsity), and the point $U$ with coordinates $(0,0)$ represents the maximal dissonance (i.e., the uncertainty). From this interpretation it becomes easy to identify the top of consonant IF pairs $\left(\mu_{j j^{\prime}}, \vartheta_{j j^{\prime}}\right)$ that fall in bottom right corner of TFU-triangle limited by vertical line from $x$-axis $x=\alpha$ and horizontal line from $y$-axis $y=\beta$. The set of consonant IF pairs are then ranked according to their Euclidean distance $d_{C_{j} C_{j^{\prime}}}^{T}$ with respect to $T$ point of coordinate $(1,0)$ defined by 


$$
d_{C_{j} C_{j^{\prime}}}^{T}=d\left((1,0),\left(\mu_{j j^{\prime}}, \vartheta_{j j^{\prime}}\right)\right)=\sqrt{\left(1-\mu_{j j^{\prime}}\right)^{2}+\vartheta_{j j^{\prime}}^{2}} .
$$

In the MCDM context only the criteria that are negatively consonant (or uncorrelated) must be kept for solving MCDM and saving computational resources because they have no (or only very low) dependency with each other, so that each uncorrelated criterion provides useful information. The set of criteria that are positively consonant (if any), called the consonant set, indicates somehow a redundancy of information between the criteria belonging to it in term of decisional behaviour. Therefore all these positively consonant criteria must be represented by only one representative criterion that will be kept in the MCDM analysis to simplify MCDM problem. Also all the criteria that are deemed strongly dissonant (if any) could be taken out of the original MCDM problem because they only introduce uncertainty in the decision-making.

\subsection{General comments on ICrA}

Although appealing at the first glance, the classical ICrA approach induces the following comments:

- The IF values $\mu_{j j^{\prime}}$, and $\vartheta_{j j^{\prime}}$ can be easily interpreted in the belief function framework. Indeed, the belief and plausibility of (positive) consonance between criteria $C_{j}$ and $C_{j^{\prime}}$ can be directly linked to the values $\mu_{j j^{\prime}}$ and $\vartheta_{j j^{\prime}}$ by taking $\operatorname{Bel}_{j j^{\prime}}(\theta)=\mu_{j j^{\prime}}$ and $\operatorname{Pl}_{j j^{\prime}}(\theta)=1-\vartheta_{j j^{\prime}}$. Moreover $U_{j j^{\prime}}(\theta)=$ $\mathrm{Pl}_{j j^{\prime}}(\theta)-\operatorname{Bel}_{j j^{\prime}}(\theta)=1-\vartheta_{j j^{\prime}}-\mu_{j j^{\prime}}$ represents the dissonance (the uncertainty about the correlation) of the criteria $C_{j}$ and $C_{j^{\prime}}$. Here the proposition $\theta$ means: the criteria $C_{j}$ and $C_{j^{\prime}}$ are totally positively consonant (i.e., totally correlated) and the frame of discernment is defined as $\Theta \triangleq\{\theta, \bar{\theta}\}$, where $\bar{\theta}$ means: the criteria $C_{j}$ and $C_{j^{\prime}}$ are totally negatively consonant (uncorrelated). From this, one can define any BBA $m_{j j^{\prime}}(\theta), m_{j j^{\prime}}(\bar{\theta})$ and $m_{j j^{\prime}}(\theta \cup \bar{\theta})$ of $2^{\Theta}$ by:

$$
\begin{gathered}
m_{j j^{\prime}}(\theta)=\mu_{j j^{\prime}}, \\
m_{j j^{\prime}}(\bar{\theta})=\vartheta_{j j^{\prime}}, \\
m_{j j^{\prime}}(\theta \cup \bar{\theta})=1-\mu_{j j^{\prime}}-\vartheta_{j j^{\prime}} .
\end{gathered}
$$

- The construction of $\mu_{j j^{\prime}}$ and $\vartheta_{j j^{\prime}}$ proposed in the classical ICrA is disputable because it is only based on counting the valid " $>$ " or " $<$ " inequalities but it doesn't exploit how bigger and how smaller the scores values are in each comparison done in the construction of the Inter-Criteria Matrix K. Therefore the construction of $\mu_{j j^{\prime}}$ and $\vartheta_{j j^{\prime}}$ is actually only a very crude method to estimate IF pairs.

- The construction of the Inter-Criteria Matrix $\mathbf{K}$ is in fact not unique as reported in [33]. This will yield different results in general.

- The exploitation of the ICrA method depends on the choice of $\alpha$ and $\beta$ thresholds that will impact the final result.

- The classical ICrA method cannot deal directly with imprecise or missing score values. 


\section{A new ICrA method based on belief functions}

We present in this section a new ICrA method, called BF-ICrA for short, based on belief functions that circumvents most of the aforementioned drawbacks of classical ICrA. Here we show how to get more precisely the Inter-Criteria Belief Matrix and how to exploit it for MCDM simplification.

\subsection{Construction of BBA matrix from the score matrix}

From any non-zero score matrix $S=\left[S_{i j}\right]$, we can construct the $N \times N$ BBA matrix $M=\left[m_{i j}().\right]$ as follows:

$$
\begin{gathered}
m_{i j}\left(A_{i}\right)=\operatorname{Bel}_{i j}\left(A_{i}\right), \\
m_{i j}\left(\overline{A_{l}}\right)=\operatorname{Bel}_{i j}\left(\overline{A_{l}}\right)=1-\operatorname{Pl}_{i j}\left(A_{i}\right), \\
m_{i j}\left(A_{i} \cup \overline{A_{l}}\right)=\mathrm{Pl}_{i j}\left(A_{i}\right)-\operatorname{Bel}_{i j}\left(A_{i}\right) .
\end{gathered}
$$

Assuming $A_{\max }^{j} \neq 0$ and $A_{\text {min }}^{j} \neq 0$, we take:

$$
\begin{aligned}
& \text { If } A_{\text {max }}^{j}=0 \text { then } \operatorname{Bel}_{i j}\left(A_{i}\right)=0 \text { and if } A_{\text {min }}^{j}=0 \text { then } \operatorname{Pl}_{i j}\left(A_{i}\right)=1 \text {, } \\
& \operatorname{Bel}_{i j}\left(A_{i}\right) \triangleq \operatorname{Sup}_{j}\left(A_{i}\right) / A_{\text {max }}^{j}, \\
& \operatorname{Bel}_{i j}\left(\overline{A_{l}}\right) \triangleq \operatorname{Inf}_{j}\left(A_{i}\right) / A_{\text {min }}^{j}
\end{aligned}
$$

where $A_{\text {max }}^{j} \triangleq \max _{i} \operatorname{Sup}_{j}\left(A_{i}\right)$ and $A_{\text {min }}^{j} \triangleq \min _{i} \operatorname{Inf}_{j}\left(A_{i}\right)$ and with

$$
\begin{gathered}
\operatorname{Sup}_{j}\left(A_{i}\right) \triangleq \sum_{k \in\{1, \ldots, M\} \mid S_{k j} \leq S_{i j}}\left|S_{i j}-S_{k j}\right|, \\
\operatorname{Inf}_{j}\left(A_{i}\right) \triangleq \sum_{k \in\{1, \ldots, M\} \mid S_{k j} \geq S_{i j}}\left|S_{i j}-S_{k j}\right| .
\end{gathered}
$$

The entire justification of these formulas can be found in our previous works [3]. For another criterion $C_{j^{\prime}}$ and the $j^{\prime}$-th column of the score matrix we will obtain another set of BBA values $m_{i j^{\prime}}($.$) . Applying this method for each column of the$ score matrix we are able to compute the BBA matrix $M=\left[m_{i j}().\right]$ each component of which is in fact a triplet $\left(m_{i j}\left(A_{i}\right), m_{i j}\left(\overline{A_{l}}\right), m_{i j}\left(A_{i} \cup \overline{A_{l}}\right)\right)$ of BBA values in $[0,1]$ such that $m_{i j}\left(A_{i}\right)+\left(\overline{A_{l}}\right)+m_{i j}\left(A_{i} \cup \overline{A_{l}}\right)=1$ for all $i=1, \ldots, M$ and $j=1, \ldots, N$.

\subsection{Construction of Inter-Criteria Matrix from BBA matrix}

The next step of BF-ICrA approach is the construction of the $N \times N$ Inter-Criteria Matrix $K=\left[K_{j j^{\prime}}\right]$ from $M \times N$ BBA matrix $M=\left[m_{i j}().\right]$, where elements $K_{j j^{\prime}}$ corresponds to the $\operatorname{BBA}\left(m_{j j^{\prime}}(\theta), m_{j j^{\prime}}(\bar{\theta}), m_{j j^{\prime}}(\theta \cup \bar{\theta})\right)$ about positive consonance $\theta$, negative consonance $\bar{\theta}$, and uncertainty between criteria $C_{j}$ and $C_{j j^{\prime}}$, respectively. The principle of construction of the triplet $K_{j j^{\prime}}=\left(m_{j j^{\prime}}(\theta), m_{j j^{\prime}}(\bar{\theta}), m_{j j^{\prime}}(\theta \cup \bar{\theta})\right)$ is based on two steps that will be detailed in the sequel:

Step 1. For each alternative $A_{i}$, we first compute the BBA $\left(m_{i j}\left(A_{i}\right), m_{i j}\left(\overline{A_{l}}\right), m_{i j}\left(A_{i} \cup \overline{A_{l}}\right)\right)$ for any two criteria $j, j^{\prime} \in\{1, \ldots, N\}$.

Step 2. The BBA $\left(m_{j j^{\prime}}(\theta), m_{j j^{\prime}}(\bar{\theta}), m_{j j^{\prime}}(\theta \cup \bar{\theta})\right)$ is then obtained by the combinations of the $M$ BBA $m_{j j^{\prime}}^{i}$. 
We present the details of each step of BF-ICrA method.

Step 1. Construction of BBA $\boldsymbol{m}_{\boldsymbol{j} j^{\prime}}^{\boldsymbol{i}}($.$) . The mass of belief m_{j j^{\prime}}^{i}(\theta)$ represents the degree of agreement between the BBA $m_{i j}($.$) and m_{j j^{\prime}}($.$) for the alternative A_{i}$, and $m_{j j^{\prime}}^{i}(\bar{\theta})$ represents the degree of disagreement between $m_{i j}($.$) and m_{j j^{\prime}}($.$) . The$ mass $m_{j j^{\prime}}^{i}(\theta \cup \bar{\theta})$ is the degree of uncertainty about the agreement (or disagreement) between $m_{i j}($.$) and m_{j j^{\prime}}($.$) for the alternative A_{i}$, The calculation of $m_{j j^{\prime}}^{i}(\theta)$ could be envisaged in several manners.

The first manner would consist to consider the degree of conflict [28] $k_{j j^{\prime}}^{i} \triangleq \sum_{X, Y \subseteq \Theta \mid X \cap Y=\varnothing} m_{i j}(X) m_{i j^{\prime}}(Y)$ and consider the Bayesian BBA $m_{j j^{\prime}}^{i}(\theta)=1-k_{j j^{\prime}}^{i}, m_{j j^{\prime}}^{i}(\bar{\theta})=k_{j j^{\prime}}^{i} \quad$ and $m_{j j^{\prime}}^{i}(\theta \cup \bar{\theta})=0$. Instead of using Shafer's conflict, the second manner would consist of using a normalized distance $d_{j j^{\prime}}^{i}=d\left(m_{i j}, m_{j j^{\prime}}\right)$ to measure the closeness between $m_{j j^{\prime}}$ and $\left.m_{j j^{\prime}}\right)$ and then consider the Bayesian BBA modelling defined by $m_{j j^{\prime}}^{i}(\theta)=1-d_{j j^{\prime}}^{i}$, $m_{j j^{\prime}}^{i}(\bar{\theta})=d_{j j^{\prime}}^{i}$ and $m_{j j^{\prime}}^{i}(\theta \cup \bar{\theta})=0$. These two manners however are not very satisfying because they always set to zero the degree of uncertainty between the agreement and disagreement of the BBA, and the second manner depends also on the choice of the distance metric. So, we propose a more appealing third manner of the BBA modelling of $m_{j j^{\prime}}^{i}(\theta), m_{j j^{\prime}}^{i}(\bar{\theta})$ and $m_{j j^{\prime}}^{i}(\theta \cup \bar{\theta})$. For this, we consider two Sources of Evidences (SoE) indexed by $j$ and $j^{\prime}$ providing the BBA $m_{i j}($. and $m_{j j^{\prime}}($.$) defined on the simple FoD \left\{A_{i}, \overline{A_{l}}\right\}$ and denoted $m_{i j}=\left[m_{i j}\left(A_{i}\right), m_{i j}\left(\overline{A_{l}}\right), m_{i j}\left(A_{i} \cup \overline{A_{l}}\right)\right] \quad$ and $\quad m_{j j^{\prime}}=\left[m_{j j^{\prime}}\left(A_{i}\right), m_{j j^{\prime}}\left(\overline{A_{l}}\right)\right.$, $\left.m_{j j^{\prime}}\left(A_{i} \cup \bar{A}_{l}\right)\right]$. We also denote $\Theta=\{\theta, \bar{\theta}\}$ the FoD about the relative state of the two SoE, where $\theta$ means that the two SoE agree, $\bar{\theta}$ means that they disagree and $\theta \cup \bar{\theta}$ means that we don't know. Then the BBA modelling is based on the important remarks:

- Two SoE are in total agreement if both commit their maximum belief mass to the element $A_{i}$ or to element $\bar{A}_{i}$. So they perfectly agree if $m_{i j}\left(A_{i}\right)=m_{i j^{\prime}}\left(A_{i}\right)=$ 1 , or if $m_{i j}\left(\bar{A}_{i}\right)=m_{i j^{\prime}}\left(\bar{A}_{i}\right)=1$. Therefore the pure degree of agreement (or positive consonance according Atanassov's terminology) between two sources is modeled by

$$
m_{j j^{\prime}}^{i}(\theta)=m_{i j}\left(A_{i}\right) m_{i j^{\prime}}\left(A_{i}\right)+m_{i j}\left(\bar{A}_{i}\right) m_{i j^{\prime}}\left(\bar{A}_{i}\right) \text {. }
$$

- Two SoE are in total disagreement if each one commits its maximum mass of belief to one element and the other to its opposite, that is if one has $m_{i j}\left(A_{i}\right)=1$ and $m_{i j^{\prime}}\left(\bar{A}_{i}\right)=1$, or if $m_{i j}\left(\bar{A}_{i}\right)=1$ and $m_{i j^{\prime}}\left(A_{i}\right)$ Hence the pure degree of disagreement (or negative consonance according Atanassov's terminology) between two sources is modelled by

$$
m_{j j^{\prime}}^{i}(\bar{\theta})=m_{i j}\left(A_{i}\right) m_{i j^{\prime}}\left(\bar{A}_{i}\right)+m_{i j}\left(\bar{A}_{i}\right) m_{i j^{\prime}}\left(A_{i}\right) .
$$

- All possible remaining products between components of $m_{i j}$ and $m_{i j^{\prime}}$ reflect the part of uncertainty we have about the SoE (i.e., we don't know if they agree or disagree). Hence the degree of uncertainty between the two sources is modelled by 


$$
m_{j j^{\prime}}^{i}(\theta \cup \bar{\theta})=m_{i j}\left(A_{i}\right) m_{i j^{\prime}}\left(A_{i} \cup \overline{A_{l}}\right)+m_{i j}\left(\bar{A}_{i}\right) m_{i j^{\prime}}\left(A_{i} \cup \overline{A_{l}}\right)+
$$

$+m_{i j}\left(A_{i} \cup \overline{A_{l}}\right) m_{i j^{\prime}}\left(A_{i}\right)+m_{i j}\left(A_{i} \cup \overline{A_{l}}\right) m_{i j^{\prime}}\left(\overline{A_{l}}\right)+m_{i j}\left(A_{i} \cup \overline{A_{l}}\right) m_{i j^{\prime}}\left(A_{i} \cup \overline{A_{l}}\right)$.

By construction $m_{j j^{\prime}}^{i}()=.m_{j^{\prime} j}^{i}($.$) , hence this BBA modeling permits to build$ a set of $M$ symmetrical Inter-Criteria Belief Matrices (ICBM) $K^{i}=\left[K_{j j^{\prime}}^{i}\right]$ of dimension $N \times N$ relative to each alternative $A_{i}$ whose components $K_{j j^{\prime}}^{i}$ correspond to the triplet of BBA values $m_{j j^{\prime}}^{i}=\left(m_{j j^{\prime}}^{i}(\theta), m_{j j^{\prime}}^{i}(\bar{\theta}), m_{j j^{\prime}}^{i}(\theta \cup \bar{\theta})\right)$ modeling the belief of agreement and of disagreement between $C_{j}$ and $C_{j^{\prime}}$ based on $A_{i}$. One has also $m_{j j^{\prime}}^{i}(\theta), m_{j j^{\prime}}^{i}(\bar{\theta}), \quad m_{j j^{\prime}}^{i}(\theta \cup \bar{\theta}) \in[0,1]$ and $m_{j j^{\prime}}^{i}(\theta),+(\bar{\theta})+m_{j j^{\prime}}^{i}(\theta \cup \bar{\theta})=1$ (because

$$
\left(m_{i j}\left(A_{i}\right)+m_{i j}\left(\bar{A}_{i}\right)+m_{i j}\left(A_{i} \cup \bar{A}_{l}\right)\right)\left(m_{i j^{\prime}}\left(A_{i}\right)+m_{i j^{\prime}}\left(\overline{A_{l}}\right)+\right.
$$

$\left.\left.m_{i j^{\prime}}\left(A_{i} \cup \overline{A_{l}}\right)\right)=1 \times 1=1\right)$. This BBA construction can be easily extended for modeling the agreement, disagreement and uncertainty of $n>2$ criteria $C_{j_{1}}, \ldots, C_{j_{n}}$ altogether if needed by taking

$$
\begin{aligned}
& m_{j_{1}, \ldots, j_{n}}^{i}(\theta)=\prod_{k=1}^{n} m_{i j_{k}}\left(A_{i}\right)+\prod_{k=1}^{n} m_{i j_{k}}\left(\bar{A}_{i}\right),
\end{aligned}
$$

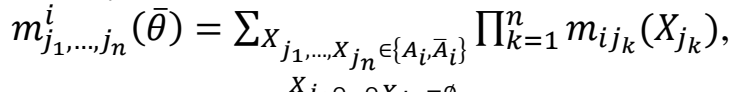

$$
\begin{aligned}
& X_{j_{1} \cap \ldots \cap X_{j_{n}}}=\varnothing \\
& m_{j_{1}, \ldots, j_{n}}^{i}(\theta \cup \bar{\theta})=1-m_{j_{1}, \ldots, j_{n}}^{i}(\theta)-m_{j_{1}, \ldots, j_{n}}^{i}(\bar{\theta}) \text {. }
\end{aligned}
$$

Step 2. Construction of BBA $\boldsymbol{m}_{\boldsymbol{j} j^{\prime}}($.$) (fusion step). Once all the BBAs$ $m_{j j^{\prime}}^{i}().(i=1, \ldots, M)$ are calculated one combines them to get the component $K_{j j^{\prime}}=\left(m_{j j^{\prime}}(\theta), m_{j j^{\prime}}(\bar{\theta}), m_{j j^{\prime}}(\theta \cup \bar{\theta})\right)$ of ICBM $K=\left[K_{j j^{\prime}}\right]$. This fusion step can be done in many ways depending on the combination rule chosen by the user. If the number of alternatives $M$ is not too large we recommend to combine the BBAs $m_{j j^{\prime}}^{i}$.) with PCR6 fusion rule [30, Vol. 3] because of known deficiencies of Dempster's rule. If $M$ is too large to prevent PCR6 working on computer, we can just use the simple averaging rule of combination in these high dimensional MCDM problems. Simple Matlab code for PCR6 rule can be found in [42] for convenience.

The computational complexity of BF-ICrA is of course higher than the complexity of ICrA because it makes a more precise evaluation of local and global inter-criteria belief matrices with respect to Intuitionistic Fuzzy inter-criteria matrices of ICrA. The overall reduction of the computational burden of the original MCDM problem thanks to BF-ICrA depends highly on the problem under concern, the complexity and cost to evaluate each criteria involved in it, as well as the number of redundant criteria identified by BF-ICrA method.

\section{Simplification of original MCDM thanks to BF-ICrA}

Once the global Inter-Criteria Belief Matrix

$$
K=\left[K_{j j^{\prime}}=\left(m_{j j^{\prime}}(\theta), m_{j j^{\prime}}(\bar{\theta}), m_{j j^{\prime}}(\theta \cup \bar{\theta})\right)\right]
$$

is calculated, we need to identify and cluster the criteria that are in strong agreement, in strong disagreement, and those on which we are uncertain. For identifying the 
criteria that are in very strong agreement, we evaluate the distance of each component of $K_{j j^{\prime}}$ with the BBA representing the best agreement state and characterized by the specific BBA $m_{T}(\theta)=1$. We use the index $T$ in the notation $m_{T}($.$) to refer that the$ agreement is true, and $F$ in $m_{F}($.$) to specify that the agreement is false. From a$ similar approach we can also identify, if we want, the criteria that are in very strong disagreement using the distance of $m_{j j^{\prime}}($.$) with respect to the BBA representing the$ best disagreement state characterized by the specific BBA $m_{F}(\bar{\theta})=1$. As alternative of Jousselme's distance [37], we use the $d_{\mathrm{BI}}(.,$.$) distance based on Belief Interval$ (BI) [36] because it is a good method for measuring the distance $d\left(m_{1}, m_{2}\right)$ between the two BBAs $m_{1}($.$) and m_{2}($.$) (here m_{1}()=.m_{j j^{\prime}}($.$) , and m_{2}()=.m_{T}($.$) , or$ $\left.m_{2}()=.m_{F}().\right)$ over the same FoD. It is defined by

$$
\left(m_{1}, m_{2}\right) \triangleq \sqrt{N_{c} \cdot \sum_{X \in 2^{\Theta}} d_{W}^{2}\left(\mathrm{BI}_{1}(X), \mathrm{BI}_{2}(X)\right)},
$$

where the belief intervals are defined by $\mathrm{BI}_{1}(X) \triangleq\left[\operatorname{Bel}_{1}(X), \mathrm{Pl}_{1}(X)\right]$ and $\mathrm{BI}_{2}(X) \triangleq$ $\left[\mathrm{Bel}_{2}(X), \mathrm{Pl}_{2}(X)\right]$ and computed from $m_{1}($.$) and m_{2}($.$) thanks to formula (1).$ $d_{W}\left(\mathrm{BI}_{1}(X), \mathrm{BI}_{2}(X)\right)$ is Wasserstein's distance between intervals calculated by

$$
d_{W}\left(\left[a_{1}, b_{1}\right],\left[a_{2}, b_{2}\right]\right)=\sqrt{\left[\frac{a_{1}+b_{1}}{2}-\frac{a_{2}+b_{2}}{2}\right]^{2}},
$$

and $N_{c}=\frac{1}{2^{|\Theta|}-1}$ is a factor to get $d_{\mathrm{BI}}\left(m_{1}, m_{2}\right) \in[0,1]$.

Because all criteria that are in strong agreement somehow contain redundant (correlated) information and behave similarly from decision-making standpoint, we propose to simplify the original MCDM problem by keeping in the MCDM only criteria that are non-redundant. The remaining criteria can be eventually weighted by their degree of importance reflecting the number of different criteria that are in agreement through this BF-ICrA approach.

For instance, if one has a seven criteria MCDM problem and if criteria $C_{1}, C_{2}$, and $C_{3}$ are in strong agreement we will only select one remaining criterion among $\left\{C_{1}, C_{2}, C_{3}\right\}$ and we give it a weight of $\omega_{1}+\omega_{2}+\omega_{3}$. Moreover if $C_{4}$ and $C_{5}$ are in strong agreement also we will only select one remaining criterion among $\left\{C_{4}, C_{5}\right\}$ and we give it a weight of $\omega_{4}+\omega_{5}$, and we will use the weight $\omega_{6}$ for $C_{6}$, and $\omega_{7}$ for $C_{7}$ Hence the original MCDM problem will reduce to a four simplified MCDM problem that can be solved using BF-TOPSIS method already presented in details in [3] and in [8], or with AHP [4] if one prefers, or with any other chosen method that the system-designer may prefer.

The strategy for selecting the most representative criterion among a set of redundant criteria is not unique and depends mainly on the cost necessary (i.e. human efforts, data mining, computational resources, etc.) for getting the values of the score matrix of the problem under concern. The least costly criteria may be a good option of selection.

In some MCDM problems the adjustment of weighting factors is necessary and the simplified MCDM allows only to reduce the computational complexity of the original problem. But in particular MCDM problems, it is not always very judicious to readjust the weights of criteria for the simplified MCDM from the weights of 
original MCDM problem because it seems more reasonable to choose them once the the simplified MCDM is determined as done in the example of Section 6.2.

In [38] we provided simple detailed examples for BF-ICrA where we selected the representative criterion as being the one with smallest index. So in the aforementioned example the simplified MCDM problem will reduce to a $M \times 4$ MCDM problem involving only four criteria $C_{1}, C_{4}, C_{6}$, and $C_{7}$.

The BF-ICrA method proposed in this work allows also, in principle, to make a refined analysis (if necessary) based on IC matrices $K_{j j^{\prime}}^{i}$ about the origin of disagreement between criteria with respect to each alternative $A_{i}$ in order to identify the potential inconsistencies in original MCDM problem. This aspect is not developed in this paper and has been left for future investigations. It is worth mentioning that the analysis of the number of redundant criteria versus time improvements that could be proposed as an effective measure of performance of this approach depends highly of the application under consideration and the difficulty (and cost) to get the value of each criteria.

\section{Two applications of BF-ICrA}

In this section we present two applications of the BF-ICrA approach. The first one is for Global Positioning System (GPS) Global Surveying Problems (GSP) presented in [39], and the second one is for the car selection problem.

\subsection{Application of BF-ICrA for the GPS surveying problem}

GPS surveying is an NP-hard problem. For designing Global Positioning System surveying network a given set of earth points must be observed consecutively. The survey cost is the sum of the distances to go from one point to another one. This kind of problems is hard to be solved with traditional numerical methods. Here we use BFICrA to analyse an Ant Colony Optimization (ACO) algorithm developed to provide near-optimal solutions for Global Positioning System surveying problem.

GPS satellites continuously transmit radio signals to the Earth while orbiting it. A receiver, with unknown position on Earth, has to detect and convert the signals received from all of the satellites into useful measurements. These measurements would allow a user to compute a three-dimensional coordinate position: location of the receiver. Any GPS observation is proven to have biases, hence, in order to survey an appropriate combination of measurement processing strategies must be used to minimize their effect on the positioning results. Differencing data collected simultaneously from two or more GPS receivers to several GPS satellites allows to eliminate or significantly reduce most of the biases. The GPS network can be defined as set of stations $\left(a_{1}, a_{2}, \ldots, a_{n}\right)$, which are co-ordinated by placing receivers $\left(X_{1}, X_{2}, \ldots\right)$ on them to determine sessions $\left(a_{1} a_{2}, a_{1} a_{3}, a_{2} a_{3}, \ldots\right)$ among them. The problem is to search for the best order in which these sessions can be organized to give the best schedule. Thus, the schedule can be defined as a sequence of sessions to be observed consecutively. The solution is represented by linear graph with weighted edges. The nodes represent the stations and the edges represent the moving 
cost. The objective function of the problem is the cost of the solution which is the sum of the costs (time) to move from one point to another one. $C(V)=\sum C\left(a_{i}, a_{j}\right)$, where $a_{i} a_{j}$ is a session in solution $V$. For example if the number of points (stations) is 4 , a possible solution is $V=\left(a_{1}, a_{3}, a_{2}, a_{4}\right)$ and it can be represented by linear graph $a_{1} \rightarrow a_{3} \rightarrow a_{2} \rightarrow a_{4}$. The moving costs are as follows: $C\left(a_{1}, a_{3}\right), C\left(a_{3}, a_{2}\right)$, $C\left(a_{2}, a_{4}\right)$. Thus the cost of the solution is $C(V)=C\left(a_{1}, a_{3}\right)+C\left(a_{3}, a_{2}\right)+$ $C\left(a_{2}, a_{4}\right)$. In practice, determining how each GPS receiver should be moved between stations to be surveyed in an efficient manner taking into account some important factors such as time, cost etc. The problem is to search for the best order, with respect to the time, in which these sessions can be observed to give the cheapest schedule or to minimize $C(V)$. The initial data is a cost matrix, which represents the cost (time, or distance) of moving a receiver from one point to another. Solving such problems - GSPs - to optimality requires a very high computational time. Therefore, metaheuristic methods are used to provide near-optimal solutions for large networks within acceptable amount of computational effort. In this paper, we consider the MaxMin Ant System (MMAS) meta-heuristic [40] and we present it briefly in the next subsection.

Real ants foraging for food lay down quantities of pheromone (chemical cues) marking the path that they follow. An isolated ant moves essentially at random but an ant encountering a previously laid pheromone will detect it and decide to follow it with high probability and thereby reinforce it with a further quantity of pheromone. The repetition of the above mechanism represents the auto-catalytic behaviour of real ant colony where the more the ants follow a trail, the more attractive that trail becomes.

The ACO Algorithm uses a colony of artificial ants that behave as cooperative agents in a mathematical space where they are allowed to search and reinforce pathways (solutions) in order to find the optimal ones. The problem is represented by graph and the ants walk on the graph to construct solutions. The solution is represented by path in the graph. After initialization of the pheromone trails, ants construct feasible solutions, starting from random nodes, then the pheromone trails are updated. At each step ants compute a set of feasible moves and select the best one (according to some probabilistic rules) to carry out the rest of the tour. The transition probability $p_{i j}$, to choose the node $j$ when the current node is $i$, is based on the heuristic information $\eta_{i j}$ and pheromone trail level $\tau_{i j}$ of the move, $i, j=1, \ldots, n$ :

$$
p_{i j}=\frac{\tau_{i j}^{\alpha} \eta_{i j}^{\beta}}{\sum_{k \in \text { Unused }} \tau_{i k}^{\alpha} \eta_{i k}^{\beta}} .
$$

The higher value of the pheromone and the heuristic information, the more profitable is to select this move and resume the search. In the beginning, the initial pheromone level is set to a small positive constant value $\tau_{0}$ and then ants update this value after completing the construction stage. ACO Algorithms adopt different criteria to update the pheromone level.

In our implementation we use MAX-MIN Ant System (MMAS) [40, 41] which is one of the best ant approaches. In MMAS the main is using fixed upper bound $\tau_{\max }$ and lower bound $\tau_{\min }$ of the pheromone trails. Thus accumulation of big amount of 
pheromone by part of the possible movements and repetition of same solutions is partially prevented.

The aim of using only one solution is to make solution elements, which frequently occur in the best found solutions, get large reinforcement. Pheromone trail update is given by:

$$
\tau_{i j} \leftarrow \rho \tau_{i j}+\Delta \tau_{i j}
$$

where

$$
\Delta \tau_{i j}=\left\{\begin{array}{cr}
1 / C\left(V_{\text {best }}\right) \text { if }(i, j) \in \text { best solution, } \\
0 & \text { otherwise, }
\end{array}\right.
$$

and $V_{\text {best }}$ is the iteration best solution and $i, j=1, \ldots, n$.

To avoid stagnation of the search, the range of possible pheromone value on each movement is limited to an interval $\left[\tau_{\min }, \tau_{\max }\right] . \tau_{\max }$ is an asymptotic maximum of $\tau_{i j}$ and $\tau_{\max }=1 /(1-\rho) C\left(V^{*}\right)$, while $\tau_{\min }=0.087 \tau_{\max }$. Where $V^{*}$ is the optimal solution, but it is unknown, therefore we use $V_{\text {best }}$ instead of $V^{*}$.

When all ants have completed their solutions, the pheromone level is updated by applying the global update rule. Only the pheromone corresponding to the best found solution is increased by the similar to the MMAS way. The global update rule is intended to provide a greater amount of pheromone on the paths of the best solution. It is a kind of intensification of the search around the best found solution. We use heuristic information equals to one over the cost of the session.

Here, we analyse the experimental results obtained using MMAS Algorithm. For this, we use real data from Malta and Seychelles GPS networks composed of 38 sessions and 71 sessions respectively denoted $\mathrm{GSP}_{1}$ and $\mathrm{GSP}_{2}$. We use also six larger test problems range from 100 to 443 sessions denoted $\mathrm{GSP}_{3}, \ldots, \mathrm{GSP}_{8}$. The results are obtained by performing 30 independent runs, for every experiment. The details of our MMAS implementation are given in [43]. So in our GSP example we consider eight GSP criteria $C_{i}=\mathrm{GSP}_{i}, i=1, \ldots, 8$, and six average costs as results $A_{1}, \ldots, A_{6}$, where $A_{1}$ is the cost average for the first 5 runs, $A_{2}$ the cost average for the first 10 runs, $A_{3}$ for the first 15 runs), $\ldots$, and finally $A_{6}$ for all the 30 runs. Table 1 shows the values of averaged costs obtained for this problem. It corresponds to the transpose of the score matrix $\mathbf{S}$.

Hence in this problem $M=6$ and $N=8$, and $S=\left[S_{i j}\right]$ is a $6 \times 8$ score matrix. Based on classical ICrA approach, one gets the following IC matrices (for presentation convenience and due to typesetting column width, we decompose at present the IC matrix $K=\left[K_{j j^{\prime}}=\left(K_{j j^{\prime}}^{\mu}, K_{j j^{\prime}}^{v}\right)\right]$ into two distinct matrices $K^{\mu}=\left[K_{j j^{\prime}}^{\mu}\right]$ and $\left.K^{v}=\left[K_{j j^{\prime}}^{v}\right]\right)$ : 


$$
\begin{aligned}
& \begin{array}{llllllll}
C_{1} & C_{2} & C_{3} & C_{4} & C_{5} & C_{6} & C_{7} & C_{8}
\end{array} \\
& C_{1}\left[\begin{array}{cccccccc}
1 & 0.60 & 0.27 & 0.67 & 0.73 & 0.67 & 0.33 & 0.87
\end{array}\right] \\
& \begin{array}{l|llllllll}
C_{2} & 0.60 & 1 & 0.27 & 0.80 & 0.73 & 0.53 & 0.47 & 0.73
\end{array} \\
& \begin{array}{l|lllllllll}
C_{3} & 0.27 & 0.27 & 1 & 0.07 & 0 & 0.20 & 0.40 & 0.13 \\
\hline
\end{array}
\end{aligned}
$$

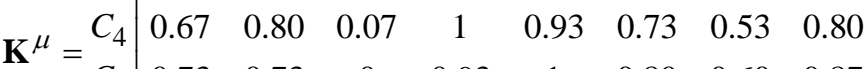

$$
\begin{aligned}
& \begin{array}{l|lllllllll}
C_{5} & 0.73 & 0.73 & 0 & 0.93 & 1 & 0.80 & 0.60 & 0.87
\end{array} \\
& \begin{array}{l|llllllll}
C_{6} & 0.67 & 0.53 & 0.20 & 0.73 & 0.80 & 1 & 0.67 & 0.80
\end{array} \\
& \begin{array}{l|lllllllll}
C_{7} & 0.33 & 0.47 & 0.40 & 0.53 & 0.60 & 0.67 & 1 & 0.47
\end{array} \\
& C_{8}\left[\begin{array}{llllllll}
0.87 & 0.73 & 0.13 & 0.80 & 0.87 & 0.80 & 0.47 & 1
\end{array}\right]
\end{aligned}
$$

Table 1. Transpose of the score matrix $S=\left[S_{i j}\right]$ of GSP problem

\begin{tabular}{|c|c|c|c|c|c|c|}
\hline $\mathrm{GSP}$ Criteria & $A_{1}$ & $A_{2}$ & $A_{3}$ & $A_{4}$ & $A_{5}$ & $A_{6}$ \\
\hline$C_{1}=\mathrm{GSP}_{1}$ & 899.00 & 898.00 & 898.33 & 898.50 & 899.40 & 899.50 \\
\hline$C_{2}=\mathrm{GSP}_{2}$ & 916.40 & 915.60 & 922.47 & 924.80 & 924.72 & 922.07 \\
\hline$C_{3}=\mathrm{GSP}_{3}$ & 41336.40 & 41052.40 & 40991.93 & 40935.90 & 40832.20 & 40910.60 \\
\hline$C_{4}=\mathrm{GSP}_{4}$ & 3244.80 & 3303.30 & 3327.00 & 3344.55 & 3345.60 & 3341.93 \\
\hline$C_{5}=\mathrm{GSP}_{5}$ & 1656.20 & 1660.80 & 1663.93 & 1664.95 & 1666.96 & 1665.90 \\
\hline$C_{6}=\mathrm{GSP}_{6}$ & 1673.60 & 1683.50 & 1690.73 & 1688.75 & 1690.24 & 1692.67 \\
\hline$C_{7}=\mathrm{GSP}_{7}$ & 3420.00 & 3430.70 & 3433.13 & 3426.85 & 3429.44 & 3428.57 \\
\hline$C_{8}=\mathrm{GSP}_{8}$ & 3758.20 & 3755.70 & 3758.73 & 3760.50 & 3760.80 & 3765.80 \\
\hline
\end{tabular}

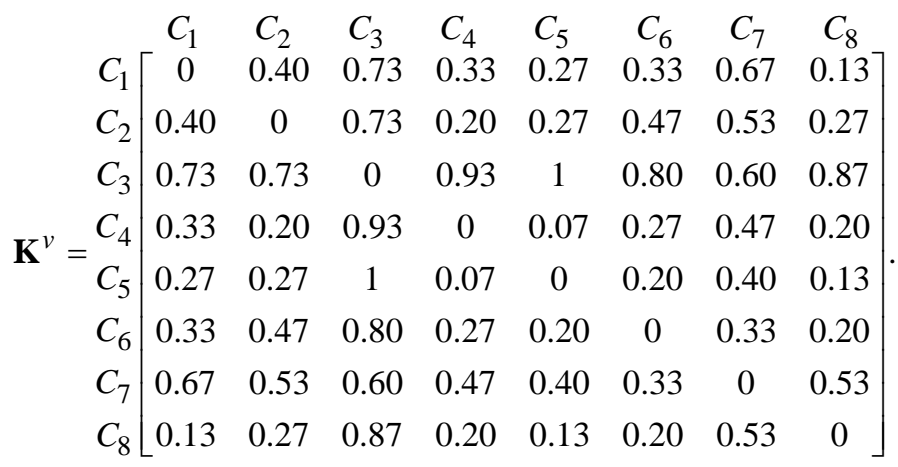

The element $K_{j j^{\prime}}^{\mu}$ of matrix $K^{\mu}$ expresses the degree of agreement between criteria $C_{j}=\mathrm{GSP}_{j}$ and $C_{j^{\prime}}=\mathrm{GSP}_{j^{\prime}}$, whereas the element $K_{j j^{\prime}}^{v}$ of matrix $K^{\mu}$ expresses the degree of disagreement between $C_{j}=\mathrm{GSP}_{j}$ and $C_{j^{\prime}}=\mathrm{GSP}_{j^{\prime}}$. Based on these results, one sees that ACO algorithm performs similarly for $\mathrm{GSP}_{2}, \mathrm{GSP}_{4}, \mathrm{GSP}_{5}$ and $\mathrm{GSP}_{8}$, because they are all in high agreement. Indeed $\mu_{j j^{\prime}}$ values for $j, j^{\prime} \in\{2,4,5,8\}$ are quite high (greater than 70\%). They are GPS networks with different numbers of sessions, but may have a similar structure, therefore, the value of agreement is high. For other networks, we can conclude that they have very different structure. What is worth noting is that there appears also a strong agreement of $\mathrm{GSP}_{1}$ with $\mathrm{GSP}_{8}$ because $\mu_{18}=0.87$. But because $\mathrm{GSP}_{8}$ is also in strong agreement with $\mathrm{GSP}_{2}, \mathrm{GSP}_{4}, \mathrm{GSP}_{5}$ and with $\mathrm{GSP}_{1}$ it is logically expected that GSP 1 should be also in agreement with $\mathrm{GSP}_{2}, \mathrm{GSP}_{4}, \mathrm{GSP}_{5}$, which is unfortunately 
not the case based on this classical ICrA. This example points out some inconsistency of ICrA result because of the too crude method of estimation of the degree of agreement and disagreement between criteria based on IFS.

Now if we consider the same example with the same score matrix $\mathbf{S}$, built from Table 1, we obtain the following IC Belief matrices (for presentation convenience, the ICBM $K_{j j^{\prime}}=\left(m_{j j^{\prime}}(\theta), m_{j j^{\prime}}(\bar{\theta}), m_{j j^{\prime}}(\theta \cup \bar{\theta})\right)$, is decomposed into three matrices $K(\theta)=\left[K_{j j^{\prime}}^{\theta}=m_{j j^{\prime}}(\theta)\right], \quad K(\bar{\theta})=\left[K_{j j^{\prime}}^{\bar{\theta}}=m_{j j^{\prime}}(\bar{\theta})\right]$, and $K(\theta \cup \bar{\theta})=$ $\left.\left[K_{j j^{\prime}}^{\theta \cup \bar{\theta}}=1-m_{j j^{\prime}}(\theta)-m_{j j^{\prime}}(\bar{\theta})\right]\right)$ :

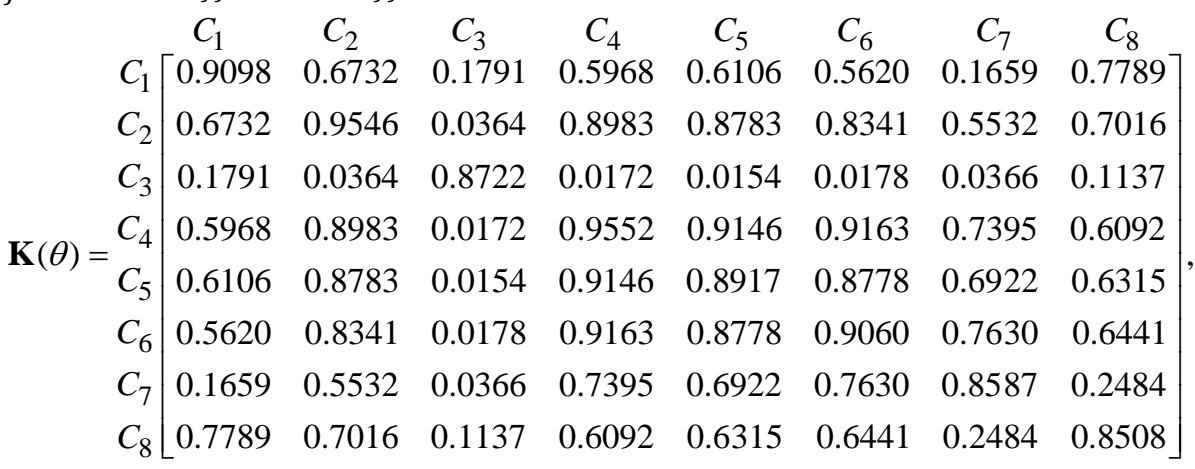

$$
\begin{aligned}
& \begin{array}{llllllll}
C_{1} & C_{2} & C_{3} & C_{4} & C_{5} & C_{6} & C_{7} & C_{8}
\end{array} \\
& C_{1}\left[\begin{array}{llllllll}
0.0207 & 0.1941 & 0.5385 & 0.2578 & 0.1757 & 0.2117 & 0.5335 & 0.0399
\end{array}\right] \\
& \begin{array}{l|llllllll}
C_{2} & 0.1941 & 0.0166 & 0.8323 & 0.0486 & 0.0298 & 0.0513 & 0.1808 & 0.0682
\end{array} \\
& \begin{array}{l|lllllllll}
C_{3} & 0.5385 & 0.8323 & 0.0117 & 0.9002 & 0.8754 & 0.8548 & 0.7062 & 0.5486
\end{array} \\
& \left.\mathbf{K}(\bar{\theta})=\begin{array}{l|llllllll}
C_{4} & 0.2578 & 0.0486 & 0.9002 & 0.0187 & 0.0216 & 0.0204 & 0.0606 & 0.1193 \\
C_{5} & 0.1757 & 0.0298 & 0.8754 & 0.0216 & 0.0170 & 0.0201 & 0.0558 & 0.0832 \\
C_{6} & 0.2117 & 0.0513 & 0.8548 & 0.0204 & 0.0201 & 0.0154 & 0.0390 & 0.0726 \\
C_{7} & 0.5335 & 0.1808 & 0.7062 & 0.0606 & 0.0558 & 0.0390 & 0.0110 & 0.3495 \\
C_{8} & 0.0399 & 0.0682 & 0.5486 & 0.1193 & 0.0832 & 0.0726 & 0.3495 & 0.0100
\end{array}\right] .
\end{aligned}
$$

\begin{tabular}{|c|c|c|c|c|c|c|c|c|}
\hline \multicolumn{9}{|c|}{$\mathbf{D}(\theta)=\left[D_{i j^{\prime}}=d_{\mathrm{BI}}\left(m_{j j^{\prime}}, m_{T}\right)\right]=$} \\
\hline$C_{1}$ & $\begin{array}{c}C_{1} \\
\lceil 0.0590\end{array}$ & $\begin{array}{c}C_{2} \\
0.2633\end{array}$ & $\begin{array}{c}C_{3} \\
0.6845\end{array}$ & $\begin{array}{c}C_{4} \\
0.3331\end{array}$ & $\begin{array}{c}C_{5} \\
0.2892\end{array}$ & $\begin{array}{c}C_{6} \\
0.3314\end{array}$ & $\begin{array}{c}C_{7} \\
0.6893\end{array}$ & $\begin{array}{c}C_{8} \\
0.1406\end{array}$ \\
\hline$C_{2}$ & 0.2633 & 0.0321 & 0.8987 & 0.0767 & 0.0803 & 0.1135 & 0.3230 & 0.1950 \\
\hline$C_{3}$ & 0.6845 & 0.8987 & 0.0774 & 0.9418 & 0.9306 & 0.9192 & 0.8381 & 0.7241 \\
\hline$C_{4}$ & 0.3331 & 0.0767 & 0.9418 & 0.0326 & 0.0566 & 0.0552 & 0.1706 & 0.2668 \\
\hline$C_{5}$ & 0.2892 & 0.0803 & 0.9306 & 0.0566 & 0.0679 & 0.0770 & 0.1958 & 0.2404 \\
\hline$C_{6}$ & 0.3314 & 0.1135 & 0.9192 & 0.0552 & 0.0770 & 0.0592 & 0.1494 & 0.2293 \\
\hline$C_{7}$ & 0.6893 & 0.3230 & 0.8381 & 0.1706 & 0.1958 & 0.1494 & 0.0849 & 0.5626 \\
\hline$C_{8}$ & 0.1406 & 0.1950 & 0.7241 & 0.2668 & 0.2404 & 0.2293 & 0.5626 & 0.0892 \\
\hline
\end{tabular}

From ICBM $K(\theta)$ and $K(\bar{\theta})$, we compute the matrix $D(\theta)$ of distance of $m_{j j^{\prime}}($.$) to the full agreement state with BBA m_{F}(\theta)=1$ based on $d_{\mathrm{BI}}($.$) distance.$ We get the following distances to full agreement: 
The element $D_{j j^{\prime}}$ represents the agreement distance between $C_{j}$, and $C_{j^{\prime}}$, the lower the better. From the values of elements of $D(\theta)$ matrix one sees clearly that ACO performs similarly for GSP2, GSP4 and GSP5 because distances $D_{24}, D_{25}$, and $D_{45}$ are very small. Also we see that GSP6 is also in good agreement with GSP4 and GSP5 but is relatively less in agreement with GSP2 because $D_{26}=0.1135$. As we see there is no inconsistency in this new BF-ICrA method with respect to what provides classical ICrA because with BF-ICrA we have a much better and precise estimation of degrees of agreement and disagreement between criteria for making the analysis thanks to a proper belief functions modelling.

\subsection{Application of BF-ICrA for the car selection problem}

Let's consider another concrete problem related to car selection. Suppose one has a limited budget of $12000 €$ and one wants to buy a new car based on multiple criteria. A set of potential cars under $12 \mathrm{~K} €$ that present interest with respect to some criteria is obtained initially from a search on the web.

How to apply BF-ICrA to simplify the selection process, and how to make the final choice of the car to buy?

Here we consider a set of ten small urban cars $\left\{A_{1}, A_{2}, \ldots, A_{10}\right\}$ as follows:

$A_{1}==$ DACIA SANDERO SCe 75;

$A_{2}==$ RENAULT CLIO TCe 75;

$A_{3}==$ SUZUKI CELERIO 1.0 VVT Avantage;

$A_{4}==$ FORD KA+ Ka+ 1.270 ch S\&S Essential;

$A_{5}==$ MITSUBISHI SPACE STAR 1.0 MIVEC 71;

$A_{6}==$ KIA PICANTO 1.0 essence MPi 67 ch BVM5 Motion;

$A_{7}=$ HYUNDAI I10 1.0 66 BVM5 Initia;

$A_{8}=$ CITROEN C1 VTi 72 S\&S Live;

$A_{9}=$ TOYOTA AYGO 1.0 VVT-i x;

$A_{10}=$ PEUGEOT 108 VTi 72ch S\&S BVM5 Like.

We consider the following seventy criteria related to price, dimensions, engine and consumption of the car for making the choice of the best car to buy:

$C_{1}$ is the price $(€)$;

$C_{2}$ is the length $(\mathrm{mm})$;

$C_{3}$ is the height $(\mathrm{mm})$;

$C_{4}$ is the width without mirror (mm);

$C_{5}$ is the wheelbase (mm);

$C_{6}$ is the max loading volume (1);

$C_{7}$ is the tank capacity (l);

$C_{8}$ is the unloaded weight $(\mathrm{kg})$;

$C_{9}$ is the cylinder volume $\left(\mathrm{cm}^{3}\right)$;

$C_{10}$ is the acceleration $0-100 \mathrm{~km} / \mathrm{h}(\mathrm{s})$;

$C_{11}$ is the max speed $(\mathrm{km} / \mathrm{h})$;

$C_{12}$ is the power $(\mathrm{kW})$;

$C_{13}$ is the horse power (HP);

$C_{14}$ is the mixed consumption (l per $100 \mathrm{~km}$ ); 
$C_{15}$ is the extra-urban consumption (1 per $100 \mathrm{~km}$ );

$C_{16}$ is the urban consumption (l per $100 \mathrm{~km}$ );

$C_{17}$ is the $\mathrm{CO}_{2}$ emission level $(\mathrm{g} / \mathrm{km})$

The score matrix $S=\left[S_{i j}\right]$ is built from information extracted from car-makers technical characteristics available on the world wide web site https://automobile.choisir.com/comparateur/voitures-neuves. For the chosen cars, the corresponding original score matrix is given by

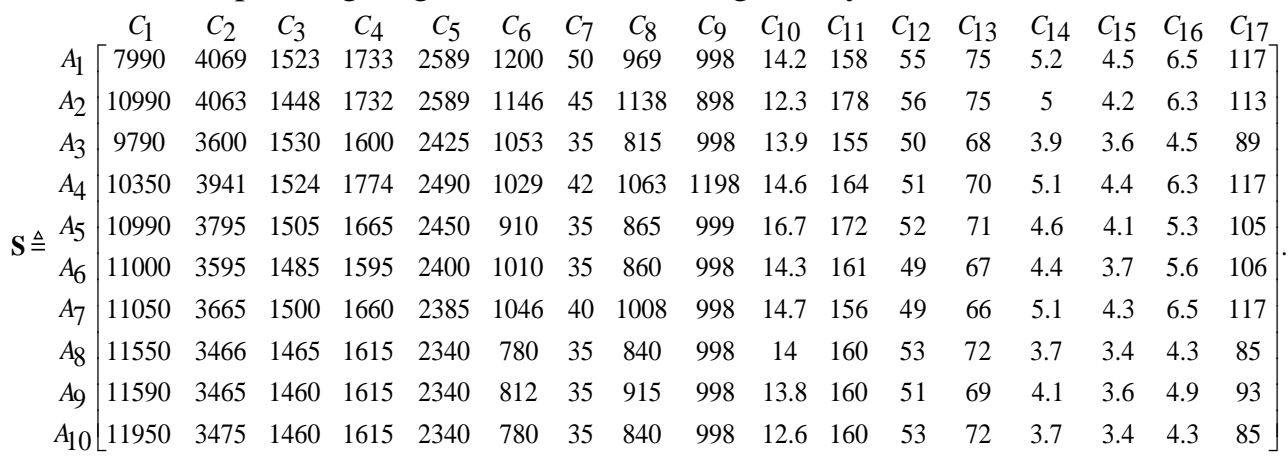

For criteria $C_{1}, C_{4}, C_{8}$, and $C_{14}$ to $C_{17}$ we consider that smaller is better. For other criteria larger is better. To make the preference order homogeneous in the score matrix, we multiply values of columns $C_{1}, C_{4}, C_{8}$, and $C_{14}$ to $C_{17}$ by -1 so that our MCDM problem is described by a modified score matrix with homogeneous preference order ("larger is better") for each column before applying the BF-ICrA method.

After applying BF-ICrA method (with PCR6 fusion rule in Step 2) we obtain the following IC Belief matrices $K(\theta)=\left[m_{j j^{\prime}}(\theta)\right], K(\bar{\theta})=\left[m_{j j^{\prime}}(\bar{\theta})\right]$, and $K(\theta \cup \bar{\theta})$ (the ICBM $K(\theta \cup \bar{\theta})$ is obtained from $K(\theta)$ and $K(\bar{\theta})$ by taking $\left.K(\theta \cup \bar{\theta})=\left[K_{j j^{\prime}}^{\theta \cup \bar{\theta}}=1-m_{j j^{\prime}}(\theta)-m_{j j^{\prime}}(\bar{\theta})\right]\right)$

$\begin{array}{lllllllllllllllll}C_{1} & C_{2} & C_{3} & C_{4} & C_{5} & C_{6} & C_{7} & C_{8} & C_{9} & C_{10} & C_{11} & C_{12} & C_{13} & C_{14} & C_{15} & C_{16} & C_{17}\end{array}$ $C_{1}\left[\begin{array}{lllllllllllllllll}0.7610 & 0.6456 & 0.6005 & 0.0722 & 0.6689 & 0.6518 & 0.6988 & 0.1152 & 0.3728 & 0.3624 & 0.1885 & 0.3273 & 0.3528 & 0.0836 & 0.0593 & 0.0981 & 0.1024\end{array}\right.$ $\begin{array}{llllllllllllllllll}C_{2} & 0.6456 & 0.8905 & 0.4994 & 0.0281 & 0.8716 & 0.7718 & 0.8635 & 0.0579 & 0.1022 & 0.2123 & 0.5406 & 0.6069 & 0.6062 & 0.0572 & 0.0411 & 0.0746 & 0.0760\end{array}$ \begin{tabular}{l|lllllllllllllllll}
$C_{3}$ & 0.6005 & 0.4994 & 0.8352 & 0.2913 & 0.4792 & 0.5196 & 0.4764 & 0.4315 & 0.5934 & 0.5300 & 0.1100 & 0.1676 & 0.2137 & 0.2570 & 0.1837 & 0.3016 & 0.2678
\end{tabular} $C_{4} \begin{array}{llllllllllllllllll} & 0.0722 & 0.0281 & 0.2913 & 0.8523 & 0.0403 & 0.0899 & 0.0402 & 0.7553 & 0.0688 & 0.1874 & 0.0941 & 0.1048 & 0.1066 & 0.7690 & 0.7849 & 0.7521 & 0.7520\end{array}$ $\begin{array}{llllllllllllllllll}C_{5} & 0.6689 & 0.8716 & 0.4792 & 0.0403 & 0.8730 & 0.7741 & 0.8602 & 0.0684 & 0.0588 & 0.1916 & 0.5275 & 0.6167 & 0.6093 & 0.0889 & 0.0650 & 0.1098 & 0.1148\end{array}$ $\begin{array}{llllllllllllllllll}C_{6} & 0.6518 & 0.7718 & 0.5196 & 0.0899 & 0.7741 & 0.8063 & 0.7533 & 0.0863 & 0.1126 & 0.2059 & 0.3050 & 0.3777 & 0.4096 & 0.0528 & 0.0493 & 0.0528 & 0.0572\end{array}$ $\begin{array}{llllllllllllllllll}C 7 & 0.6988 & 0.8635 & 0.4764 & 0.0402 & 0.8602 & 0.7533 & 0.9492 & 0.0455 & 0.1989 & 0.1959 & 0.5019 & 0.6660 & 0.6169 & 0.0964 & 0.0783 & 0.1028 & 0.1371\end{array}$ \begin{tabular}{l|lllllllllllllllll}
$C_{8}$ & 0.1152 & 0.0579 & 0.4315 & 0.7553 & 0.0684 & 0.0863 & 0.0455 & 0.8060 & 0.2495 & 0.3144 & 0.0877 & 0.1262 & 0.1472 & 0.7398 & 0.7042 & 0.7632 & 0.7424
\end{tabular}

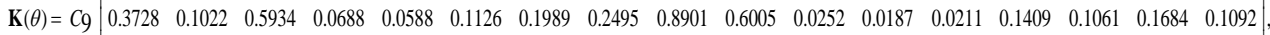
$C_{10} \begin{array}{lllllllllllllllllll}0.3624 & 0.2123 & 0.5300 & 0.1874 & 0.1916 & 0.2059 & 0.1959 & 0.3144 & 0.6005 & 0.7484 & 0.1268 & 0.0447 & 0.0500 & 0.1628 & 0.1087 & 0.2057 & 0.1720\end{array}$

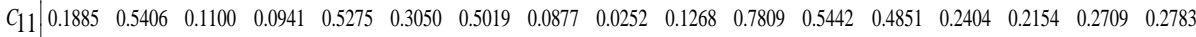
\begin{tabular}{l|llllllllllllllllll}
$C_{12}$ & 0.3273 & 0.6069 & 0.1676 & 0.1048 & 0.6167 & 0.3777 & 0.6660 & 0.1262 & 0.0187 & 0.0447 & 0.5442 & 0.7845 & 0.7665 & 0.2940 & 0.2388 & 0.3387 & 0.3693
\end{tabular}

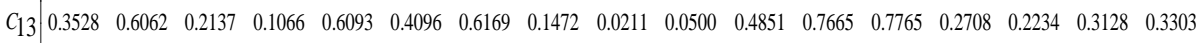
$C_{14} \begin{array}{lllllllllllllllllll}0.0836 & 0.0572 & 0.2570 & 0.7690 & 0.0889 & 0.0528 & 0.0964 & 0.7398 & 0.1409 & 0.1628 & 0.2404 & 0.2940 & 0.2708 & 0.8921 & 0.8678 & 0.8986 & 0.9066\end{array}$ \begin{tabular}{l|lllllllllllllllll}
$C_{15}$ & 0.0593 & 0.0411 & 0.1837 & 0.7849 & 0.0650 & 0.0493 & 0.0783 & 0.7042 & 0.1061 & 0.1087 & 0.2154 & 0.2388 & 0.2234 & 0.8678 & 0.8628 & 0.8634 & 0.8735
\end{tabular} $\begin{array}{llllllllllllllllll}C_{16} & 0.0981 & 0.0746 & 0.3016 & 0.7521 & 0.1098 & 0.0528 & 0.1028 & 0.7632 & 0.1684 & 0.2057 & 0.2709 & 0.3387 & 0.3128 & 0.8986 & 0.8634 & 0.9141 & 0.9168\end{array}$

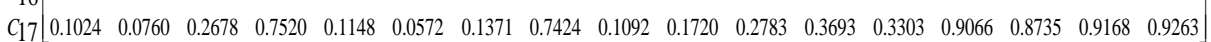


$\begin{array}{lllllllllllllllll}C_{1} & C_{2} & C_{3} & C_{4} & C_{5} & C_{6} & C_{7} & C_{8} & C_{9} & C_{10} & C_{11} & C_{12} & C_{13} & C_{14} & C_{15} & C_{16} & C_{17}\end{array}$

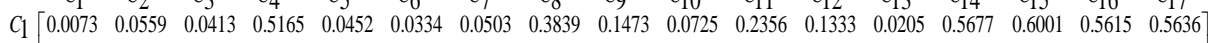
$\begin{array}{llllllllllllllllll}C_{2} & 0.0559 & 0.0232 & 0.2781 & 0.8331 & 0.0262 & 0.0469 & 0.0527 & 0.7364 & 0.2368 & 0.2329 & 0.1259 & 0.1136 & 0.1168 & 0.8256 & 0.8285 & 0.8187 & 0.8251\end{array}$ $\begin{array}{llllllllllllllllll}C_{3} & 0.0413 & 0.2781 & 0.0199 & 0.4166 & 0.2692 & 0.1566 & 0.3470 & 0.2421 & 0.0132 & 0.0353 & 0.5231 & 0.4722 & 0.4097 & 0.5242 & 0.5658 & 0.5041 & 0.5527\end{array}$ \begin{tabular}{l|lllllllllllllllll}
$C_{4}$ & 0.5165 & 0.8331 & 0.4166 & 0.0164 & 0.7815 & 0.6067 & 0.8499 & 0.0324 & 0.3318 & 0.2199 & 0.5259 & 0.5691 & 0.5472 & 0.0623 & 0.0396 & 0.0880 & 0.0972
\end{tabular} \begin{tabular}{l|lllllllllllllllll}
$C_{5}$ & 0.0452 & 0.0262 & 0.2692 & 0.7815 & 0.0222 & 0.0407 & 0.0434 & 0.6918 & 0.3119 & 0.2453 & 0.1233 & 0.0963 & 0.1065 & 0.7609 & 0.7695 & 0.7530 & 0.7560
\end{tabular} \begin{tabular}{l|lllllllllllllllll|}
$C_{6}$ & 0.0334 & 0.0469 & 0.1566 & 0.6067 & 0.0407 & 0.0153 & 0.0724 & 0.5777 & 0.3117 & 0.2007 & 0.2177 & 0.1845 & 0.1727 & 0.7491 & 0.7309 & 0.7676 & 0.7671
\end{tabular} \begin{tabular}{l|lllllllllllllllll}
$C_{7}$ & 0.0503 & 0.0527 & 0.3470 & 0.8499 & 0.0434 & 0.0724 & 0.0175 & 0.8074 & 0.1993 & 0.2931 & 0.1976 & 0.1074 & 0.1416 & 0.8036 & 0.8032 & 0.8152 & 0.7871
\end{tabular}

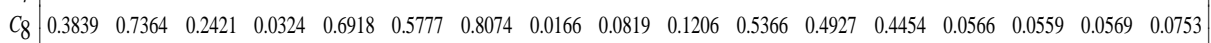

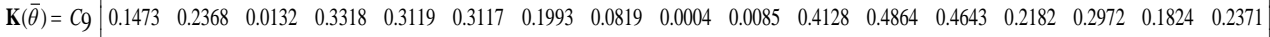
$\begin{array}{llllllllllllllllll}C_{10} & 0.0725 & 0.2329 & 0.0353 & 0.2199 & 0.2453 & 0.2007 & 0.2931 & 0.1206 & 0.0085 & 0.0045 & 0.2490 & 0.4253 & 0.4127 & 0.2898 & 0.3474 & 0.2535 & 0.3006\end{array}$ \begin{tabular}{l|llllllllllllllllll}
$C_{11}$ & 0.2356 & 0.1259 & 0.5231 & 0.5259 & 0.1233 & 0.2177 & 0.1976 & 0.5366 & 0.4128 & 0.2490 & 0.0096 & 0.0790 & 0.1087 & 0.4037 & 0.3761 & 0.4053 & 0.4043
\end{tabular} \begin{tabular}{l|lllllllllllllllllll}
$C_{12}$ & 0.1333 & 0.1136 & 0.4722 & 0.5691 & 0.0963 & 0.1845 & 0.1074 & 0.4927 & 0.4864 & 0.4253 & 0.0790 & 0.0157 & 0.0179 & 0.3934 & 0.4018 & 0.3820 & 0.3633
\end{tabular} \begin{tabular}{l|lllllllllllllllll|}
$C_{13}$ & 0.1205 & 0.1168 & 0.4097 & 0.5472 & 0.1065 & 0.1727 & 0.1416 & 0.4454 & 0.4643 & 0.4127 & 0.1087 & 0.0179 & 0.0163 & 0.4209 & 0.4233 & 0.4092 & 0.4013
\end{tabular} $C_{14} \quad \begin{array}{lllllllllllllllllll}0.5677 & 0.8256 & 0.5242 & 0.0623 & 0.7609 & 0.7491 & 0.8036 & 0.0566 & 0.2182 & 0.2898 & 0.4037 & 0.3934 & 0.4209 & 0.0247 & 0.0244 & 0.0282 & 0.0271\end{array}$

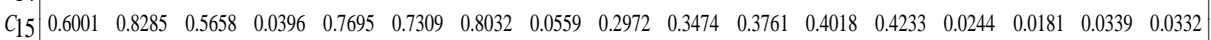
\begin{tabular}{l|lllllllllllllllll}
$C_{16}$ & 0.5615 & 0.8187 & 0.5041 & 0.0880 & 0.7530 & 0.7676 & 0.8152 & 0.0569 & 0.1824 & 0.2535 & 0.4053 & 0.3820 & 0.4092 & 0.0282 & 0.0339 & 0.0257 & 0.0272
\end{tabular} $C_{17}\left[\begin{array}{lllllllllllllllll}0.5636 & 0.8251 & 0.5527 & 0.0972 & 0.7560 & 0.7671 & 0.7871 & 0.0753 & 0.2371 & 0.3006 & 0.4043 & 0.3633 & 0.4013 & 0.0271 & 0.0332 & 0.0272 & 0.0240\end{array}\right]$

From ICBM $K(\theta)$ and $K(\bar{\theta})$ we compute the matrix $D(\theta)=\left[D_{j j^{\prime}}=\right.$ $\left.d_{\mathrm{BI}}\left(m_{j j^{\prime}}, m_{T}\right)\right]$ of distance of the BBA $m_{j j^{\prime}}$ with respect to the full agreement state having BBA $m_{\mathrm{F}}(\theta)=1$ based on $d_{\mathrm{BI}}($.$) distance. We get the following distances to$ full agreement

$\begin{array}{ccccccccccccccccc}C_{1} & C_{2} & C_{3} & C_{4} & C_{5} & C_{6} & C_{7} & C_{8} & C_{9} & C_{10} & C_{11} & C_{12} & C_{13} & C_{14} & C_{15} & C_{16} & C_{17} \\ \end{array}$ $C_{1}\left[\begin{array}{lllllllllllllllll}0.1401 & 0.2225 & 0.2434 & 0.7318 & 0.2054 & 0.2114 & 0.1901 & 0.6506 & 0.4113 & 0.3907 & 0.5493 & 0.4320 & 0.4128 & 0.7489 & 0.7766 & 0.7383 & 0.7369\end{array}\right]$ \begin{tabular}{l|lllllllllllllllll}
$C_{2}$ & 0.2225 & 0.0709 & 0.3946 & 0.9034 & $\mathbf{0 . 0 8 2 7}$ & 0.1471 & $\mathbf{0 . 0 9 7 7}$ & 0.8414 & 0.5985 & 0.5349 & 0.3081 & 0.2659 & 0.2675 & 0.8848 & 0.8945 & 0.8726 & 0.8750
\end{tabular} $\begin{array}{llllllllllllllllll}C_{3} & 0.2434 & 0.3946 & 0.1014 & 0.5689 & 0.4016 & 0.3319 & 0.4383 & 0.4161 & 0.2387 & 0.2821 & 0.7145 & 0.6605 & 0.6078 & 0.6368 & 0.6948 & 0.6039 & 0.6445\end{array}$ \begin{tabular}{l|llllllllllllllllll}
$C_{4}$ & 0.7318 & 0.9034 & 0.5689 & 0.0904 & 0.8721 & 0.7634 & 0.9054 & 0.1515 & 0.6548 & 0.5438 & 0.7242 & 0.7382 & 0.7272 & 0.1545 & 0.1370 & 0.1742 & 0.1780
\end{tabular} $\begin{array}{llllllllllllllllll}C_{5} & 0.2054 & \mathbf{0 . 0 8 2 7} & 0.4016 & 0.8721 & 0.0805 & 0.1436 & 0.9058 & 0.8146 & 0.6524 & 0.5514 & 0.3145 & 0.2537 & 0.2618 & 0.8372 & 0.8536 & 0.8225 & 0.8214\end{array}$ \begin{tabular}{l|lllllllllllllllll}
$C_{6}$ & 0.2114 & 0.1471 & 0.3319 & 0.7634 & 0.1436 & 0.1165 & 0.1673 & 0.7520 & 0.6222 & 0.5261 & 0.4767 & 0.4227 & 0.4001 & 0.8501 & 0.8432 & 0.8589 & 0.8565
\end{tabular} $\begin{array}{llllllllllllllllll}C_{7} & 0.1901 & \mathbf{0 . 0 9 7 7} & 0.4383 & 0.9054 & 0.0958 & 0.1673 & 0.0355 & 0.8820 & 0.5295 & 0.5681 & 0.3585 & 0.2302 & 0.2715 & 0.8541 & 0.8632 & 0.8565 & 0.8253\end{array}$

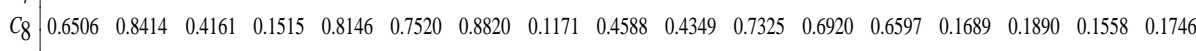

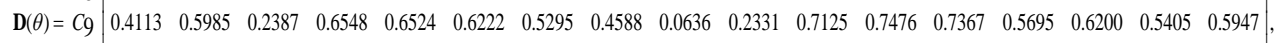
$C_{10} \begin{array}{lllllllllllllllllllll} & 0.3907 & 0.5349 & 0.2821 & 0.5438 & 0.5514 & 0.5261 & 0.5681 & 0.4349 & 0.2331 & 0.1466 & 0.5893 & 0.7070 & 0.6988 & 0.5852 & 0.6389 & 0.5466 & 0.5845\end{array}$ $C_{11} \begin{array}{llllllllllllllllllll} & 0.5493 & 0.3081 & 0.7145 & 0.7242 & 0.3145 & 0.4767 & 0.3585 & 0.7325 & 0.7125 & 0.5893 & 0.1294 & 0.2887 & 0.1331 & 0.5907 & 0.5922 & 0.5748 & 0.5704\end{array}$ \begin{tabular}{l|llllllllllllllllll}
$C_{12}$ & 0.4320 & 0.2659 & 0.6605 & 0.7382 & 0.2537 & 0.4227 & 0.2302 & 0.6920 & 0.7476 & 0.7070 & 0.2887 & 0.1292 & $\mathbf{0 . 1 4 0 3}$ & 0.5571 & 0.5907 & 0.5278 & 0.5030
\end{tabular} \begin{tabular}{l|llllllllllllllllll}
$C_{13}$ & 0.4128 & 0.2675 & 0.6078 & 0.7272 & 0.2618 & 0.4001 & 0.2715 & 0.6597 & 0.7367 & 0.6988 & 0.3331 & $\mathbf{0 . 1 4 0 3}$ & 0.1340 & 0.5819 & 0.6086 & 0.5541 & 0.5411
\end{tabular} \begin{tabular}{l|llllllllllllllllll}
$C_{14}$ & 0.7489 & 0.8848 & 0.6368 & 0.1545 & 0.8372 & 0.8501 & 0.8541 & 0.1689 & 0.5695 & 0.5852 & 0.5907 & 0.5571 & 0.5819 & 0.0705 & $\mathbf{0 . 0 8 4 2}$ & $\mathbf{0 . 0 6 8 2}$ & $\mathbf{0 . 0 6 3 2}$
\end{tabular} \begin{tabular}{l|lllllllllllllllllll}
$C_{15}$ & 0.7766 & 0.8945 & 0.6948 & 0.1370 & 0.8536 & 0.8432 & 0.8632 & 0.1890 & 0.6200 & 0.6389 & 0.5922 & 0.5907 & 0.6086 & $\mathbf{0 . 0 8 4 2}$ & 0.0849 & $\mathbf{0 . 0 9 0 2}$ & $\mathbf{0 . 0 8 4 2}$
\end{tabular} \begin{tabular}{l|lllllllllllllllll}
$C_{16}$ & 0.7383 & 0.8726 & 0.6039 & 0.1742 & 0.8225 & 0.8589 & 0.8565 & 0.1558 & 0.5405 & 0.5466 & 0.5748 & 0.5278 & 0.5541 & $\mathbf{0 . 0 6 8 2}$ & $\mathbf{0 . 0 9 0 2}$ & 0.0584 & $\mathbf{0 . 0 5 7 5}$
\end{tabular} $C_{17}\left[\begin{array}{llllllllllllllllll}0.7369 & 0.8750 & 0.6445 & 0.1780 & 0.8214 & 0.8565 & 0.8253 & 0.1746 & 0.5947 & 0.5845 & 0.5704 & 0.5030 & 0.5411 & \mathbf{0 . 0 6 3 2} & \mathbf{0 . 0 8 4 2} & \mathbf{0 . 0 5 7 5} & 0.0509\end{array}\right]$

The element $D_{j j^{\prime}}$ represents the agreement distance between $C_{j}$ and $C_{j^{\prime}}$, the lower the better. From the analysis of elements of $D_{j j^{\prime}}$ one sees clearly that criteria $C_{14}, C_{15}, C_{16}$, and $C_{17}$ are in very strong agreement and will behave very similarly for the preference ordering which is not very surprising because they are all related with energy consumption. Hence only one criteria among of these four criteria be used to simplify the MCDM car selection problem. We decide to keep only criteria $C_{16}$ (urban consumption) in simplified MCD because urban displacements will be the main use of the car. One sees clearly that $C_{5}, C_{7}$, and $C_{7}$ are also in very strong agreement and so they will behave very similarly for the preference ordering. One decides to keep only the criterion $C_{7}$ (tank capacity) which we consider more important than criteria $C_{2}$, and $C_{5}$ because it is linked to autonomy of the car. From 
BF-ICrA, one sees that tank capacity is linked with the dimensions of the car (mainly its length and wheelbase), which makes perfectly sense. Also we can note that criteria $C_{12}$, and $C_{13}$ are not too far since their distance is only 0.1403 and we can simplify a bit more the MCDM problem by taking only criterion $C_{12}$ (the power) instead of keeping $C_{12}$, and $C_{13}$. Thanks to BF-ICrA, we can simplify the original MCDM car selection problem by removing redundant criteria and keeping only those which bring useful information. So our simplified MCDM car selection problem is characterized by the following $10 \times 11$ score matrix:

\begin{tabular}{|c|c|c|c|c|c|c|c|c|c|c|c|}
\hline & $C_{1}$ & & & & & $C_{8}$ & $C_{9}$ & $C_{10}$ & $C_{11}$ & $C_{12}$ & $\begin{array}{l}16 \\
.5\end{array}$ \\
\hline & 990 & 1448 & 1732 & 1146 & 45 & 1138 & 898 & 12.3 & 178 & 56 & 6.3 \\
\hline & 790 & 1530 & 1600 & 1053 & 35 & 815 & 998 & 13.9 & 155 & 50 & 4.5 \\
\hline$A_{4}$ & 10350 & 1524 & 1774 & 1029 & 42 & 1063 & 1198 & 14.6 & 164 & 51 & 6.3 \\
\hline & 10990 & 1505 & 1665 & 910 & 35 & 865 & 999 & 16.7 & 172 & 52 & 5.3 \\
\hline & 11000 & 1485 & 1595 & 1010 & 35 & 860 & 998 & 14.3 & 161 & 49 & 5.6 \\
\hline & 11050 & 1500 & 1660 & 1046 & 40 & 1008 & 998 & 14.7 & 156 & 40 & 6.5 \\
\hline & & 1465 & 1615 & 780 & 35 & 840 & 998 & 11 & 160 & 53 & 43 \\
\hline & 1590 & 1460 & 1615 & 8 & & 0 & 998 & 13.8 & 160 & 51 & 4.9 \\
\hline & 1950 & 1460 & 1615 & 780 & 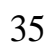 & 840 & 998 & 12.6 & 160 & 53 & 4.3 \\
\hline
\end{tabular}

From this reduced score matrix, we can apply classical MCDM techniques to find the final preference order for making final decision and selectioning the car to buy. For this, one needs to define the importance $\operatorname{imp}\left(C_{j}\right)$ of each criteria $C_{j}$ involved in the score matrix above. For simplicity, the importance of each criteria $C_{j}$ is expressed as a value in $\{1,2,3,4,5\}$, where 1 means the least important, and 5 means the most important. In this car selection example we take $p\left(C_{1}\right)=\operatorname{imp}\left(C_{16}\right)=5$, $\operatorname{imp}\left(C_{6}\right)=\operatorname{imp}\left(C_{7}\right)=4, \quad \operatorname{imp}\left(C_{10}\right)=\operatorname{imp}\left(C_{11}\right)=\operatorname{imp}\left(C_{12}\right)=3, \quad \operatorname{imp}\left(C_{8}\right)=$ $\operatorname{imp}\left(C_{9}\right)=2$ and $\operatorname{imp}\left(C_{3}\right)=\operatorname{imp}\left(C_{4}\right)=1$, which means that the price of the car and its urban consumption are the most important criteria for us, and its height and its width are the least important ones. From these importance values and after normalization, we get the following vector of relative weights of criteria

$$
w=\left[\frac{5}{33} \frac{1}{33} \frac{1}{33} \frac{4}{33} \frac{4}{33} \frac{2}{33} \frac{2}{33} \frac{3}{33} \frac{3}{33} \frac{3}{33} \frac{5}{33}\right] \text {. }
$$

When using different BF-TOPSIS methods [3, 8], we will obtain the following preference orders.

- with BF-TOPSIS1 method:

$$
A_{2}>A_{1}>A_{4}>A_{7}>A_{5}>A_{6}>A_{10}>A_{9}>A_{8}>A_{3},
$$

- with BF-TOPSIS2 method:

$$
A_{2}>A_{1}>A_{4}>A_{7}>A_{5}>A_{6}>A_{10}>A_{9}>A_{8}>A_{3},
$$

- with BF-TOPSIS3 method:

$$
A_{2}>A_{1}>A_{4}>A_{7}>A_{5}>A_{10}>A_{9}>A_{6}>A_{8}>A_{3},
$$

- with BF-TOPSIS4 method:

$$
A_{2}>A_{1}>A_{4}>A_{7}>A_{5}>A_{10}>A_{9}>A_{6}>A_{8}>A_{3} \text {. }
$$


When using classical AHP method [4], we obtain the following preference order (Here we did apply a two steps normalization of the score matrix. At first we normalize $\mathbf{S}$ according to (2) and in a second step each column is renormalized by dividing each element of the column by the sum of its elements. If we apply only first normalization step we obtain with AHP the preference order $A_{2}>A_{4}>A_{1}>A_{7}>$ $\left.A_{5}>A_{6}>A_{9}>A_{8}>A_{10}>A_{3}\right)$.

$$
A_{2}>A_{1}>A_{4}>A_{7}>A_{5}>A_{6}>A_{9}>A_{8}>A_{3}>A_{10} \text {. }
$$

From the results of the BF-TOPSIS methods and AHP (with double normalization of score matrix), one sees that $A_{2}$ car (RENAULT CLIO TCe 75) will be the best car to buy, and the car $A_{1}$ (DACIA SANDERO SCe 75) will be the second best car to buy, whereas $A_{3}$ (SUZUKI CELERIO 1.0 VVT Avantage) will be the worst one according to BF-TOPSIS or $A_{10}$ according to AHP. Because the AHP and BF-TOPSIS methods are based on very different principles it is not surprising that preference order can change in the results of the methods, but what is most important from decision-making standpoint is the stability of the order of first best solutions. In this example, the car $A_{2}$ is always the best car selection to make with BF-TOPSIS or with AHP method based on the chosen criteria involved in this MCDM problem and their importance weights.

\section{Conclusion}

In this paper we have presented a new method called BF-ICrA which helps to simplify (when it is possible) Multi-Criteria Decision-Making problems based on inter-criteria analysis and belief functions. This method is in the spirit of Atanassov's method but proposes a better construction of Inter-Criteria Matrix that fully exploits all information of the score matrix, and the closeness measure of agreement between criteria based on belief interval distance. In fact, BF-ICrA proposes a more precise and refined method for estimating the degree of agreement and disagreement between criteria which use the whole information available in the data. This BF-ICrA approach could, in theory, also deal with imprecise or missing score values using the technique presented in [8]. We have shown two concrete applications of BF-ICrA method. The first one related with the GPS surveying problem has been addressed in order to overcome the potential inconsistencies of the results generated by the classical ICrA method. Instances containing from 38 to 443 sessions have been solved using MMAS algorithm and we did compare the performance of ACO algorithms applied to eight GPS networks. Our results show that ACO can provide fast near-optimal solution for observing GPS networks, and could help to improve the services based on GPS networks. From this new Inter-Criteria Analysis we are able to identify some relations and dependences between the considered eight GPSs and MMAS algorithm performance.

In our second application, we have shown how a typical (not so simple) multicriteria car selection problem can be addressed and solved by this BF-ICrA method coupled with BF-TOPSIS methods. This shows the usefulness and potential of this new technique to solve MCDM problems. 
Acknowledgements: This paper is partially supported by the National Scientific Fund of Bulgaria under Grant DFNI DN12/5 and by Grant No BG05M2OP001-1.001-0003, financed by the Science and Education for Smart Growth Operational Program and co-financed by the European Union through the European structural and Investment funds.

\section{References}

1. B arzilai, J., B. Golany. AHP Rank Reversal, Normalization and Aggregation Rules. INFOR, Vol. 32, 1994, No 2, pp. 57-63.

2. P a v l i c i c, D. Normalization Affects the Results of MADM Methods. - Yugoslav J. of Operations Research, Vol. 11, 2001, No 2, pp. 251-265.

3. Dezert, J., D. Han, H. Y in. A New Belief Function Based Approach for Multi-Criteria Decision-Making Support. - In: Proc. of Information Fusion Conference, 2016.

4. S a a t y, T. The Analytic Hierarchy Process. McGraw-Hill, 1980.

5. W a ng, X., E. Triantaphyllou. Ranking Irregularities When Evaluating Alternatives by Using Some ELECTRE Methods. - Omega, Vol. 36, 2008, No 1.

6. H w a n g, C. L., K. Y o o n. Multiple Attribute Decision Making. - In: Lecture Notes in Economics and Math. Syst. Vol. 186. Berlin, Springer-Verlag, 1981.

7. La i, Y. J., T. Y. Li u, C. L. H w a n g. TOPSIS for MODM. - European Journal of Operational Research, Vol. 76, 1994, No 3, pp. 486-500.

8. D e z e r t, J., D. H a n, J.-M. T a c n e t. Multi-Criteria Decision-Making with Imprecise Scores and BF-TOPSIS. - In: Proc. of Information Fusion Conference, Proc. of Fusion, 2017.

9. A t a n a s sov, K., D. Mavrov, V. A t a n a s s ova. Intercriteria Decision Making. A New Approach for Multicriteria Decision Making, Based on Index Matrices and Intuitionistic Fuzzy Sets. - Issues in IFS and Generalized Nets, Vol. 11, 2014, pp. 1-8.

10. At a n a s sov, K., V. At a n a s ova, G. Gluh chev. InterCriteria Analysis: Ideas and Problems. - Notes on IFS, Vol. 21, 2015, No 1, pp. 81-88.

11. A t a n a s s o v, K., et al. An Approach to a Constructive Simplification of Multiagent Multicriteria Decision Making Problems via Intercriteria Analysis. - Compt. Rend. Acad. bulg. Sci., Vol. 70, 2017, No 8.

12. A t a $\mathrm{n}$ a s s o v, K. On Intuitionistic Fuzzy Sets Theory. Springer, 2012.

13. T o d i n o v a, S., et al. Blood Plasma Thermograms Dataset Analysis by Means of Inter Criteria and Correlation Analyses for the Case of Colorectal Cancer. - Int. J. of BIO Automation, Vol. 20, 2016, No 1, pp 115-124.

14. K r u m o v a, S., et al. InterCriteria Analysis of Calorimetric Data of Blood Serum Proteome. - In: Bioch. et Biophys. Acta, Gen. Subjects, 1861, 2017.

15. Zaharieva, B., et al. InterCriteria Decision Making Approach for Behterev's DISEASE ANALYSIS. - Int. J. of Bioautom, Vol. 22, 2018, No 2.

16. Pe ncheva, T., et al. InterCriteria Analysis of Genetic Algorithm Parameters in Parameter Identification. - Notes on IFS, Vol. 21, 2015, No 2.

17. S o t i rov, S., et al. Application of the Intuitionistic Fuzzy InterCriteria Analysis Method to a Neural Network Preprocessing Procedure. - In: Proc. of 9th EUSFLAT, 2015, pp. 1559-1564.

18. R o e va, O., et al. InterCriteria Analysis of a Model Parameters Identification Using Genetic Algorithm. - In: Proc. of Federated Conf. on Computer Science and Information Systems 5, 2015, pp. 501-506.

19. A n g e l o v a, M., O. R o e v a, T. P e $\mathrm{n}$ c h e v a. InterCriteria Analysis of Crossover and Mutation Rates Relations in Simple Genetic Algorithm. - In: Proc. of Conf on Computer Sci. and Inf. Syst, Vol. 5, 2015, pp. 419-424.

20. R o e va, O., S. Fid a n o va, M. P a p r z y cki. InterCriteria Analysis of ACO and GA Hybrid Algorithms. - Stud. Comput. Intell., Vol. 610, 2016, pp. 107-126.

21. R o e v a, O., et al. InterCriteria Analysis of ACO Performance for Workforce Planning Problem. In: Studies in Comp. Intell. Vol. 795. Springer, 2019.

22. A t a n a s s o v a, V., et al., Discussion on the Threshold Values in the InterCriteria Decision Making Approach. - Notes on Intuitionistic Fuzzy Sets, Vol. 20, 2014 No 2, pp. 94-99. 
23. Doukovska, L., V. At an as sova. InterCriteria Analysis Approach in Radar Detection Threshold Analysis. - Notes on IFS, Vol. 21, 2015, No 4.

24. D o u k o v s k a, L., et al. InterCriteria Analysis Applied to EU Micro, Small, Medium and Large Enterprises. - In Proc. of 5th Int. Symp. on BMSD, 2015.

25. B u r e va, V., et al. Application of the InterCriteria Decision Making Method to Bulgarian Universities Ranking. - In: Int. Workshop on IFSs, 2015.

26. B u r e va, V., E. S o ti r o va, H. P a n y o to v. The InterCriteria Decision Making Method to Bulgarian University Ranking System. - Annual of Informatics Section, Vol. 8, 2015-2016, pp. 54-70.

27. K r a w c z a k, M., et al. Application of the InterCriteria Decision Making Method to Universities Ranking. - Adv. in Intell. Syst. and Comp., Springer, Vol. 401, 2016, pp. 365-372.

28. S h a f e r, G. A Mathematical Theory of Evidence. Princeton Press, 1976.

29. D e m p st e r, A. Upper and Lower Probabilities Induced by a Multivalued Mapping. - Ann. of Math. Stat., Vol. 38, 1967, pp. 325-339.

30. F. Smarandache, J. Dezert, Eds. Advances and Applications of DSmT for Information Fusion. American Research Press, Vol. 1-4, 2004-2015. http://www.onera.fr/staff/jean-dezert?page $=2$

31. A t a n a s s ov, K. Index Matrices: Towards an Augmented Matrix Calculus. - Springer, Cham, 2014.

32. A t a n a s s o v, K., et al. Intercriteria Analysis over Normalized Data. - In: Proc. of 8th IEEE Int. Conf. on Intelligent Syst., 2016, pp. 564-566.

33. I k o n o m o v, N., P. V a s s i l e v, O. R o e v a. ICrAData Software for InterCriteria Analysis. - Int. J. BioAutomation, Vol. 22, 2018, No 2.

34. A t a n a s s v a, V. Interpretation in the Intuitionistic Fuzzy Triangle of the Results Obtained by the InterCriteria Analysis. - In: Proc. of 16th World Congr. of IFSA, Atlantis Press, 2015.

35. A t a n a s s o v a, V., et al. Traversing and Ranking of Elements of an Intuitionistic Fuzzy Set in the Intuitionistic Fuzzy Interpretation Triangle. - Adv. in Intell. Syst. and Comp., Vol. 401, 2016, pp. 161-174.

36. H a n, D., J. D e z e r t, Y. Y a n g. New Distance Measures of Evidence Based on Belief Intervals. - Proc. of Belief, Oxford, 2014.

37. Jous selme, A.-L., D. Grenier, E. B os sé. A New Distance between Two Bodies of Evidence. - Information Fusion, Vol. 2, 2001, No 2, pp. 91-101.

38. D e z e r t, J., A. T c h a m o v a, D. H a n, J.-M. T a c n e t. Simplification of Multi-Criteria DecisionMaking Using InterCriteria Analysis and Belief Functions. - In: Proc. of Fusion 2019 Int. Conf., 2019.

39. Fid a n o v a, S., J. D e z e r t, A. T c h a m o v a. InterCriteria Analysis Based on Belief Functions for GPS Surveying Problems. - In: Proc. of Int. Symposium on INnovations in Intelligent SysTems and Applications (INISTA'19), 2019.

40. S t u t z l e, T., H. H. H o o s. MAX-MIN Ant System. - In: M. Dorigo, T. Stutzle, G. Di Caro, Eds. Future Generation Computer Systems, Vol. 16, 2000, pp. 889-914.

41. D o ri g o, M., L. M. G a m b a r d e 11 a. Ant Colony System: A Cooperative Learning Approach to the Traveling Salesman Problem. - IEEE Trans. Evol. Comput., Vol. 1, 1997, pp. 5-66.

42. S m a r a n d a c h e, F., J. D e z e r t, J.-M. T a c n e t. Fusion of Sources of Evidence with Different Importances and Reliabilities. - In: Proc. of Fusion Conf., 2010.

43. Fid a nova, S., V. At a n a s s o va, O. R o e va. Ant Colony Optimization Application to GPS Surveying Problems: InterCriteria Analysis. - In: K. Atanassov et al., Eds. Uncertainty and Imprecision in Decision Making and Decision Support: Cross-Fertilization, New Models and Applications. IWIFSGN 2016. Advances in Intelligent Systems and Computing, Springer. Vol. 559. Cham, 2018.

Received: 04.12.2019; Accepted: 07.05.2020 